\title{
Time-analyticity of Lagrangian particle trajectories in ideal fluid flow
}

\author{
VLADISLA V ZHELIG OVSKY ${ }^{1,2}$ and URIEL FRISCH \\ ${ }^{1}$ Institute of Earthquake Prediction Theory and Mathematical Geophysics of the Russian \\ Academy of Sciences, 84/32 Profsoyuznaya St., 117997 Moscow, Russian Federation \\ ${ }^{2}$ UNS, CNRS, Lab. Lagrange, OCA, CS 34229, 06304 Nice Cedex 4, France
}

(Received 15 April 2014)

It is known that the Eulerian and Lagrangian structures of fluid flow can be drastically different; for example, ideal fluid flow can have a trivial (static) Eulerian structure, while displaying chaotic streamlines. Here we show that ideal flow with limited spatial smoothness (an initial vorticity that is just a little better than continuous), nevertheless has time-analytic Lagrangian trajectories before the initial limited smoothness is lost. For proving such results we use a little-known Lagrangian formulation of ideal fluid flow derived by Cauchy in 1815 in a manuscript submitted for a prize of the French Academy. This formulation leads to simple recurrence relations among the time-Taylor coefficients of the Lagrangian map from initial to current fluid particle positions; the coefficients can then be bounded using elementary methods. We first consider various classes of incompressible fluid flow, governed by the Euler equations, and then turn to highly compressible flow governed by the Euler-Poisson equations, a case of cosmological relevance. The recurrence relation associated to the Lagrangian formulation of these incompressible and the compressible problems are so closely related that the proofs of time-analyticity are basically identical.

\section{Introduction}

G.I. Taylor (1938) in a paper on the spectrum of turbulence observed that "If the velocity of the air stream which carries the eddies is very much greater than the turbulent velocity, one may assume that the sequence of changes in $u$ [the velocity] at the fixed point are simply due to the passage of an unchanging pattern of turbulent motion over the point, ..." This Taylor hypothesis is used by experimentalists working with wind tunnels to obtain approximate information about the spatial structure of turbulent flow from the temporal variation at the fixed location of a probe.

Taylor's argument has also implications for the spatio-temporal smoothness in Eulerian coordinates: near a space-time location of non-vanishing velocity $\boldsymbol{U}$ the temporal regularity cannot be better than the spatial regularity along the direction of $\boldsymbol{U}$, as can be seen, at least heuristically, by performing a Galilean transformation with velocity $\boldsymbol{U}$. For example, if the spatial variation of the velocity is roughly as the cubic root of the distance (Hölder continuity of exponent $1 / 3$ ), then so can be expected for the temporal variation. Recently obtained weak (distributional) solutions of the three-dimensional (3D) incompressible Euler equations give precisely the same spatial and temporal regularity in Eulerian coordinates (Buckmaster et al. 2013), and the bounds for the material derivative $\partial_{t}+(\boldsymbol{v} \cdot \nabla)$ are better than the bounds for the spatial and time derivatives (Isett 2014). In Lagrangian coordinates, where we are following fluid particles, the situation is very different. As we shall see, with sufficiently regular initial conditions, the Lagrangian temporal smoothness vastly exceeds the spatial smoothness. 
It is well known that some major mathematical questions remain open for 3D incompressible flow, be it viscous (Navier-Stokes) or ideal/inviscid (Euler). However, a number of theorems state that, when the initial data are sufficiently regular, solutions with this regularity exist for some time, and are unique. All such theorems have been proved under the assumption that the initial vorticity is slightly more regular than merely continuous - for example, that the initial vorticity is Hölder-continuous, i.e., it is bounded and its spatial increments between any two points are bounded by a positive fractional power of the distance between these points. Well-known instances of such results are the all-time regularity of 2D flow (Wolibner 1933; Hölder 1933) and the finite-time regularity of 3D flow (Lichtenstein 1927). Their proofs make use of the boundedness of the velocity gradient, which is not guaranteed, even initially, when the vorticity is just continuous (such matters are discussed further in Section 2.5).

Within such and similar frameworks, we shall show in this paper that, while the assumed initial regularity persists, the Lagrangian fluid particle trajectories are analytic functions of the time.

As usual, there were precursors - foremost, and nearly two hundred years ago, Cauchy (1815). His Lagrangian formulation of the 3D incompressible Euler equations, in terms of Lagrangian invariants, provides us with a simple framework within which we can develop an elementary theory of analytic fluid particle trajectories. Cauchy's work on invariants has been almost completely forgotten in the twentieth century and little cited in the nineteenth. Hankel (1861) and Stokes $(1848,1883)$ are notorious exceptions; a detailed review of the history of Cauchy's half-forgotten result can be found in Frisch \& Villone (2014).

Stoker (1957), in the last chapter of his book on water waves, where he discussed the Lagrangian theory of free-surface flow, presented some of the work of his Courant Institute student Frederick Pohle. This work remains unpublished except in the $\mathrm{PhD}$ thesis (Pohle 1951). Pohle rediscovered the Lagrangian invariant formulation of Cauchy in the 2D case. He was not aware of Cauchy's 3D work, but pointed out briefly that his theory can be extended to three dimensions. Pohle noticed that this Lagrangian formulation leads to recurrence relations among the formal time-Taylor coefficients. He wrote them all, but used only a few, since his goal was to apply the theory to the breakup of dams. Pohle did not prove the convergence of this formal Taylor series, i.e. analyticity, but Stoker (1957) conjectured that analyticity can be proved using techniques previously used by Lichtenstein. That is precisely what we are doing here more than half a century later.

More recently, Serfati $(1992,1995 a, b)$ argued that weak spatial regularity can nevertheless be consistent with time-analyticity of Lagrangian trajectories. He proposed studying this using a Biot-Savart-type integral formulation of the Lagrangian equations and then making use of the mathematical theory of analytic functionals (a kind of infinitedimensional generalisation of functions of many complex variables). Shnirelman (2012) tackled the problem, avoiding the Biot-Savart integrals. These papers by Serfati and Shnirelman are very interesting for those with a taste for advanced mathematics. Temporal analyticity of "particle trajectories" was also proved in the recent paper (Constantin, Vicol \& Wu 2014) for equations of hydrodynamic type with the use of rather involved combinatorial relations (called ibid. "magic identities"). Such abstract proofs do not provide us with concrete ways of constructing the time-Taylor series for the motion of fluid particles.

Frisch \& Zheligovsky (2014) gave an elementary proof of analyticity that uses Cauchy's Lagrangian formulation. It was then realised that our method can be generalised, for example, to flow that is initially analytic in the space variables. Furthermore, as conjectured 
Time-analyticity of Lagrangian particle trajectories in ideal fluid flow

by Serfati (1995a,b), analyticity of Lagrangian fluid particle trajectories holds also for compressible flow. The present paper addresses all these issues. Intended primarily for fluid dynamicists, it is self-contained and does not rely on advanced mathematical techniques.

The outline of the paper is as follows. Section 2 is entirely devoted to ideal incompressible fluid flow. In Section 2.1 we present the Lagrangian formulation of the ideal fluid equations due to Cauchy. In Section 2.2 we show how it yields recurrence relations for time-Taylor coefficients of the Lagrangian map. We then prove the analyticity of fluidparticle trajectories for the simplest case: an initial vorticity field whose Fourier series is absolutely convergent (Section 2.3). The same technique is then adapted to initial vorticities that are analytic in space (Section 2.4) or Hölder-continuous (Section 2.5). In Section 2.6 we bracket the radius of convergence of these time-Taylor series more precisely: by considering an example of a Beltrami flow with integrable Lagrangian trajectories and by refining our previously obtained bounds. Section 3 is devoted to an instance of compressible ideal fluid flow of cosmological relevance. Concluding remarks are made in Section 4.

\section{Ideal incompressible fluid flow}

\subsection{The Cauchy invariants}

The starting point of Cauchy (1815) were the equations of incompressible fluid flow, namely, the momentum equation and the incompressibility condition, expressed in what is now called Eulerian coordinates. In modern notation, they are, respectively:

$$
\begin{gathered}
\partial_{t} \boldsymbol{v}+(\boldsymbol{v} \cdot \nabla) \boldsymbol{v}=-\nabla p, \\
\nabla \cdot \boldsymbol{v}=0 .
\end{gathered}
$$

Here, $\boldsymbol{v}$ is the flow velocity, $p$ pressure, $t$ time.

Cauchy transformed the equations by switching to Lagrangian coordinates, denoted here by $\boldsymbol{a}$. Let $\boldsymbol{a} \mapsto \boldsymbol{x}=\boldsymbol{x}(\boldsymbol{a}, t)$ be the Lagrangian map from the initial to the current (i.e., at time $t$ ) Eulerian position, $\boldsymbol{x}$, of a fluid particle. The map satisfies

$$
\dot{\boldsymbol{x}}=\boldsymbol{v}(\boldsymbol{x}, t), \quad \boldsymbol{x}(\boldsymbol{a}, 0)=\boldsymbol{a},
$$

where the dot denotes the Lagrangian time derivative. Since the left-hand side of (2.1) is the Lagrangian acceleration, (2.1) implies

$$
\ddot{\boldsymbol{x}}=-\nabla p=-\nabla^{\mathrm{L}} p\left(\left(\nabla^{\mathrm{L}} \boldsymbol{x}\right)^{*}\right)^{-1},
$$

where $\nabla^{\mathrm{L}}$ denotes the gradient in the Lagrangian variables and the star denotes matrix transposition. Here, we have reexpressed the Eulerian pressure gradient in terms of the Lagrangian pressure gradient, using the Jacobian matrix $\nabla^{\mathrm{L}} \boldsymbol{x}$ with entries $\nabla_{i}^{\mathrm{L}} x_{j}$. From here on, unless otherwise stated, all space and time derivatives will be Lagrangian ones.

At this point, Cauchy took the Lagrangian curl of (2.4) and then employed a deus ex machina to time-integrate the resulting equations. Our approach involves only a minor modification, which eliminates some of the magics: following Weber (1868), we rewrite (2.4) as

$$
\frac{\mathrm{d}}{\mathrm{d} t} \sum_{k=1}^{3} \dot{x}_{k} \nabla^{\mathrm{L}} x_{k}=\nabla^{\mathrm{L}}\left(\frac{1}{2}|\dot{\boldsymbol{x}}|^{2}-p\right)
$$


and take the Lagrangian curl of (2.5) to find

$$
\frac{\mathrm{d}}{\mathrm{d} t} \nabla^{\mathrm{L}} \times \sum_{k=1}^{3} \dot{x}_{k} \nabla^{\mathrm{L}} x_{k}=\frac{\mathrm{d}}{\mathrm{d} t} \sum_{k=1}^{3} \nabla^{\mathrm{L}} \dot{x}_{k} \times \nabla^{\mathrm{L}} x_{k}=0 .
$$

The expression in the middle is obtained by applying the Lagrangian curl to each term in the sum and observing that no terms with second spatial Lagrangian derivatives emerge. The importance of this will become clear later. From (2.6) follows that the middle sum is a time-independent vector. At $t=0$, by (2.3), this vector is seen to be the initial vorticity $\boldsymbol{\omega}^{\text {(init) }}=\nabla^{\mathrm{L}} \times \boldsymbol{v}^{\text {(init) }}$. Thus we obtain Cauchy's equations in Lagrangian coordinates:

$$
\begin{aligned}
\sum_{k=1}^{3} \nabla^{\mathrm{L}} \dot{x}_{k} \times \nabla^{\mathrm{L}} x_{k} & =\boldsymbol{\omega}^{(\mathrm{init})}, \\
\operatorname{det}\left(\nabla^{\mathrm{L}} \boldsymbol{x}\right) & =1 .
\end{aligned}
$$

Here, (2.8) expresses the conservation of fluid volume. The three components of the lefthand side of (2.7), which are obviously constant along any fluid particle trajectory, have been called the Cauchy invariants (see, e.g., Yakubovich \& Zenkovich 2001). Their conservation is actually the three-dimensional generalisation of the two-dimensional vorticity conservation.

As is well known, the unforced Euler equations (2.2) are invariant under Galilean transformations. More precisely, limiting ourselves to solutions for which the velocity and the pressure are space-periodic, if $(\boldsymbol{v}(\boldsymbol{x}, t), p(\boldsymbol{x}, t))$ is a solution, then $(\boldsymbol{v}(\boldsymbol{x}-\boldsymbol{U} t, t)+\boldsymbol{U}$, $p(\boldsymbol{x}-\boldsymbol{U} t, t))$ is also a solution to the same equation, for any uniform $\boldsymbol{U}$. This corresponds to changing the frame of reference to the one moving with the velocity $\boldsymbol{U}$. If, however, we try to describe the fluid from an accelerating frame, we shall have to add a spaceindependent force term. The latter is equivalent to adding to the pressure a term linear in the spatial coordinates, but this is not consistent with space periodicity. In contrast, when using Cauchy's Lagrangian equations (2.7)-(2.8), we can switch to an arbitrary accelerated frame, provided it coincides with the original frame at $t=0$ : this just adds to the Lagrangian map a time-dependent uniform vector, which drops out of (2.7)-(2.8), because all the terms involve gradients of the map. Of course, similar remarks can be made on the Eulerian vorticity formulation of the Euler equation.

\subsection{The Taylor expansion in time}

The basic Lagrangian equations (2.7)-(2.8) do not give us an explicit expression for the time derivative of either the Lagrangian map or of its gradient. Nevertheless, they yield almost immediately explicit recurrence relations for the time-Taylor coefficients of the Lagrangian map. This will be crucial for the problems tackled here. For establishing these relations it is convenient to work with the fluid particle displacement field $\boldsymbol{\xi}(\boldsymbol{a}, t) \equiv \boldsymbol{x}(\boldsymbol{a}, t)-\boldsymbol{a}$.

In terms of the displacement, the Lagrangian equations (2.7) and (2.8) take the form

$$
\begin{aligned}
& \nabla^{\mathrm{L}} \times \dot{\boldsymbol{\xi}}+\sum_{k=1}^{3} \nabla^{\mathrm{L}} \dot{\xi}_{k} \times \nabla^{\mathrm{L}} \xi_{k}=\boldsymbol{\omega}^{(\mathrm{init})} \\
& \nabla^{\mathrm{L}} \cdot \boldsymbol{\xi}+\sum_{1 \leq i<j \leq 3}\left(\left(\nabla_{i}^{\mathrm{L}} \xi_{i}\right) \nabla_{j}^{\mathrm{L}} \xi_{j}-\left(\nabla_{i}^{\mathrm{L}} \xi_{j}\right) \nabla_{j}^{\mathrm{L}} \xi_{i}\right)+\operatorname{det}\left(\nabla^{\mathrm{L}} \boldsymbol{\xi}\right)=0 .
\end{aligned}
$$

Now, we assume that the displacement can be Taylor-expanded in the time variable 
around $t=0$ for sufficiently short times:

$$
\boldsymbol{\xi}(\boldsymbol{a}, t)=\sum_{s=1}^{\infty} \boldsymbol{\xi}^{(s)}(\boldsymbol{a}) t^{s} .
$$

Here the functions $\boldsymbol{\xi}^{(s)}$ are called the (time-)Taylor coefficients. For the time being such an expansion is only formal. The existence of all the time derivatives needed for the expansion will follow from the bounds that are established in Sections 2.3 and 2.5.

Recurrence relations for the Taylor coefficients of the displacement are obtained by substituting (2.11) into (2.9) and (2.10) and gathering all the terms containing a given power $t^{s}$ :

$$
\begin{aligned}
\nabla^{\mathrm{L}} \times \boldsymbol{\xi}^{(s)}= & \boldsymbol{\omega}^{(\mathrm{init})} \delta_{1}^{s}-\sum_{\substack{1 \leq k \leq 3, 0<m<s}} \frac{m}{s} \nabla^{\mathrm{L}} \xi_{k}^{(m)} \times \nabla^{\mathrm{L}} \xi_{k}^{(s-m)} \\
= & \boldsymbol{\omega}^{(\mathrm{init})} \delta_{1}^{s}-\frac{1}{2} \sum_{\substack{1 \leq k \leq 3 \\
0<m<s}} \frac{2 m-s}{s} \nabla^{\mathrm{L}} \xi_{k}^{(m)} \times \nabla^{\mathrm{L}} \xi_{k}^{(s-m)} . \\
\nabla^{\mathrm{L}} \cdot \boldsymbol{\xi}^{(s)}= & \sum_{\substack{1 \leq i<j \leq 3 \\
0<m<s}}\left(\left(\nabla_{j}^{\mathrm{L}} \xi_{i}^{(m)}\right) \nabla_{i}^{\mathrm{L}} \xi_{j}^{(s-m)}-\left(\nabla_{i}^{\mathrm{L}} \xi_{i}^{(m)}\right) \nabla_{j}^{\mathrm{L}} \xi_{j}^{(s-m)}\right) \\
& -\sum_{\substack{i, j, k \\
l+m+n=s}} \varepsilon_{i j k}\left(\nabla_{i}^{\mathrm{L}} \xi_{1}^{(l)}\right)\left(\nabla_{j}^{\mathrm{L}} \xi_{2}^{(m)}\right) \nabla_{k}^{\mathrm{L}} \xi_{3}^{(n)} .
\end{aligned}
$$

Here $\varepsilon_{i j k}$ is the unit antisymmetric tensor and $\delta_{s^{\prime}}^{s}$ is the Kronecker symbol; the second line of (2.12) is obtained by grouping the terms for the indices $m$ and $s-m$ in the sum in the right-hand side of its first line. It is immediately seen from the two equations (2.12) and (2.13) for $s=1$ that $\boldsymbol{\xi}^{(1)}=\boldsymbol{v}^{\text {(init) }}$.

A remarkable property of (2.12)-(2.13) is that their right-hand sides involve exclusively terms that are quadratic and cubic in the gradients of the displacement and no higher derivatives. This will be crucial in proving analyticity.

If we know all the Taylor coefficients up to order $s-1,(2.12)$ and (2.13) give us the curl and the divergence of the next Taylor coefficient of order $s$. A vector field, whose curl and divergence are known, can be uniquely (for given boundary conditions) determined using the Helmholtz-Hodge decomposition (cf., e.g., Arfken \& Weber 2005). Applying it to $(2.12)-(2.13)$, we find

$$
\boldsymbol{\xi}^{(s)}=\nabla^{-2}\left(-\nabla^{\mathrm{L}} \times \mathbf{R}^{(1)}+\nabla^{\mathrm{L}} R^{(2)}\right),
$$

where $\nabla^{-2}$ denotes the inverse Laplacian in the Lagrangian coordinates (taking into account the boundary conditions), and $\mathbf{R}^{(1)}$ and $R^{(2)}$ denote the right-hand sides of (2.12) and (2.13), respectively.

\subsection{Proving analyticity: a case study using Fourier analysis}

To begin with, in Section 2.3.1, we shall prove the time-analyticity of fluid particle trajectory by an elementary Fourier method. We assume that the solution is $2 \pi$-periodic in space and that the spatial Fourier series of the initial vorticity

$$
\boldsymbol{\omega}^{(\mathrm{init})}(\boldsymbol{a})=\sum_{\mathbf{p}} \widehat{\boldsymbol{\omega}}_{\mathbf{p}} \mathrm{e}^{\mathrm{ip} \cdot \boldsymbol{a}}
$$


is absolutely convergent:

$$
\Gamma \equiv \sum_{\mathbf{p}}\left|\widehat{\omega}_{\mathbf{p}}\right|<\infty
$$

Here summation is over three-dimensional vectors $\mathbf{p}$ with integer components. Because of the absolute convergence, clearly the initial vorticity is continuous in $\boldsymbol{a}$.

First, in Section 2.3.1 we present a proof using elementary transformations of the recurrence relations (2.12)-(2.13) in the spatial Fourier space. This "elementary" proof may, however, leave the reader wondering why it is working. To prevent the trees from hiding the forest, we then present, in Section 2.3.2 another slightly more transparent albeit more advanced "normed-space" proof in which the structure of the recurrence relations is exploited to obtain bounds for the norms of the Taylor coefficients in a suitable space of functions. The second approach can and will be generalised to other normed function spaces (initial vorticities that are analytic or Hölder-continuous), but it does not yield estimates as sharp as the elementary proof.

\subsubsection{An elementary proof by transformations in Fourier space}

Upon expanding the time-Taylor coefficients of the displacement in spatial Fourier series

$$
\boldsymbol{\xi}^{(s)}(\boldsymbol{a})=\sum_{\mathbf{p}} \widehat{\boldsymbol{\xi}}_{\mathbf{p}}^{(s)} \mathrm{e}^{\mathrm{i} \mathbf{p} \cdot \boldsymbol{a}}, \quad s=1,2, \ldots,
$$

we can express the recurrence relations in the Fourier representation. Eq. (2.12) becomes then

$$
\mathbf{p} \times \widehat{\boldsymbol{\xi}}_{\mathbf{p}}^{(s)}=-\mathrm{i} \widehat{\boldsymbol{\omega}}_{\mathbf{p}} \delta_{1}^{s}-\frac{\mathrm{i}}{2} \sum_{0<m<s, \mathbf{r}} \frac{2 m-s}{s}\left(\widehat{\boldsymbol{\xi}}_{\mathbf{r}}^{(m)} \cdot \widehat{\boldsymbol{\xi}}_{\mathbf{p}-\mathbf{r}}^{(s-m)}\right)(\mathbf{r} \times(\mathbf{p}-\mathbf{r})) .
$$

Similarly, it is elementary but a bit tedious (see Appendix A) to rewrite (2.13) as

$$
\begin{aligned}
\mathbf{p} \cdot \widehat{\boldsymbol{\xi}}_{\mathbf{p}}^{(s)}= & \frac{\mathrm{i}}{2} \sum_{0<m<s, \mathbf{r}}(\mathbf{r} \times(\mathbf{p}-\mathbf{r})) \cdot\left(\widehat{\boldsymbol{\xi}}_{\mathbf{r}}^{(m)} \times \widehat{\boldsymbol{\xi}}_{\mathbf{p}-\mathbf{r}}^{(s-m)}\right) \\
& +\frac{1}{6} \sum_{\substack{n_{1}+n_{2}+n_{3}=s \\
\mathbf{r}_{1}+\mathbf{r}_{2}+\mathbf{r}_{3}=\mathbf{p}}}\left[\mathbf{r}_{1}, \mathbf{r}_{2}, \mathbf{r}_{3}\right]\left[\widehat{\boldsymbol{\xi}}_{\mathbf{r}_{1}}^{\left(n_{1}\right)}, \widehat{\boldsymbol{\xi}}_{\mathbf{r}_{2}}^{\left(n_{2}\right)}, \widehat{\boldsymbol{\xi}}_{\mathbf{r}_{3}}^{\left(n_{3}\right)}\right],
\end{aligned}
$$

where $[\cdot, \cdot, \cdot]$ denotes the triple product.

For $\mathbf{p}=0$ equations (2.18) and (2.19) are trivially satisfied. The mean (over the periodicity cell) of the velocity is easily seen to be conserved. Without loss of generality, we henceforth assume that this mean vanishes initially. Consequently, the mean velocity and the mean displacement will also vanish at any time, and thus $\widehat{\boldsymbol{\xi}}_{0}^{(s)}=0$ for all $s$.

For $\mathbf{p} \neq 0$ the coefficient $\widehat{\boldsymbol{\xi}}_{\mathbf{p}}^{(s)}$ can be uniquely determined, as we know its components normal and parallel to the wave vector $\mathbf{p}$, by virtue of (2.18) and (2.19), respectively. (In the Fourier representation, the Helmholtz-Hodge decomposition is indeed just a geometric decomposition of a vector into transverse and longitudinal components, perpendicular and parallel to the wave vector $\mathbf{p}$.) Let $\widetilde{\mathbf{R}}_{\mathbf{p}}^{\perp}$ and $\widetilde{R}_{\mathbf{p}}^{\|}$denote the right-hand side of (2.18) and (2.19), respectively. Then

$$
\widehat{\boldsymbol{\xi}}_{\mathbf{p}}^{(s)}=|\mathbf{p}|^{-2}\left(-\mathbf{p} \times \widetilde{\mathbf{R}}_{\mathbf{p}}^{\perp}+\widetilde{R}_{\mathbf{p}}^{\|} \mathbf{p}\right) .
$$

We are now using recurrence relations (2.18) and (2.19) to derive bounds for the Fourier coefficients of the time-Taylor series. Eq. (2.18) and the observation that $|2 m-s| / s \leq 1$ 
for all $0<m<s$ imply a bound for the transverse component:

$$
\left|\mathbf{p} \times \widehat{\boldsymbol{\xi}}_{\mathbf{p}}^{(s)}\right| \leq\left|\widehat{\boldsymbol{\omega}}_{\mathbf{p}}\right| \delta_{1}^{s}+\frac{1}{2} \sum_{0<m<s, \mathbf{r}}|\mathbf{r}||\mathbf{p}-\mathbf{r}|\left|\widehat{\boldsymbol{\xi}}_{\mathbf{r}}^{(m)} \cdot \widehat{\boldsymbol{\xi}}_{\mathbf{p}-\mathbf{r}}^{(s-m)}\right| .
$$

A bound for the longitudinal component follows from (2.19):

$$
\begin{aligned}
\left|\mathbf{p} \cdot \widehat{\boldsymbol{\xi}}_{\mathbf{p}}^{(s)}\right| \leq & \frac{1}{2} \sum_{m, \mathbf{r}}|\mathbf{r}||\mathbf{p}-\mathbf{r}|\left|\widehat{\boldsymbol{\xi}}_{\mathbf{r}}^{(m)} \times \widehat{\boldsymbol{\xi}}_{\mathbf{p}-\mathbf{r}}^{(s-m)}\right| \\
& +\frac{1}{6} \sum_{\substack{n_{1}+n_{2}+n_{3}=s \\
\mathbf{r}_{1}+\mathbf{r}_{2}+\mathbf{r}_{3}=\mathbf{p}}}\left|\mathbf{r}_{1}\right|\left|\mathbf{r}_{2}\right|\left|\mathbf{r}_{3}\right|\left|\widehat{\boldsymbol{\xi}}_{\mathbf{r}_{1}}^{\left(n_{1}\right)}\right|\left|\widehat{\boldsymbol{\xi}}_{\mathbf{r}_{2}}^{\left(n_{2}\right)}\right|\left|\widehat{\boldsymbol{\xi}}_{\mathbf{r}_{3}}^{\left(n_{3}\right)}\right| .
\end{aligned}
$$

We now introduce the generating function

$$
\zeta(t) \equiv \sum_{s=1}^{\infty}\left(\sum_{\mathbf{p}}|\mathbf{p}|\left|\widehat{\boldsymbol{\xi}}_{\mathbf{p}}^{(s)}\right|\right) t^{s} .
$$

From (2.23) and the bounds (2.21) and (2.22) for the transverse and longitudinal components,

$$
\begin{aligned}
\zeta(t) \leq & \sum_{s=1}^{\infty} \sum_{\mathbf{p}}\left(\left|\mathbf{p} \times \widehat{\boldsymbol{\xi}}_{\mathbf{p}}^{(s)}\right|+\left|\mathbf{p} \cdot \widehat{\boldsymbol{\xi}}_{\mathbf{p}}^{(s)}\right|\right) t^{s} \\
\leq & \sum_{\mathbf{p}}\left|\widehat{\boldsymbol{\omega}}_{\mathbf{p}}\right| t+\frac{1}{\sqrt{2}} \sum_{s=1}^{\infty} \sum_{\substack{0<m<s, \mathbf{r}}}|\mathbf{r}||\mathbf{p}-\mathbf{r}|\left|\widehat{\boldsymbol{\xi}}_{\mathbf{r}}^{(m)}\right|\left|\widehat{\boldsymbol{\xi}}_{\mathbf{p}-\mathbf{r}}^{(s-m)}\right| t^{s} \\
& +\frac{1}{6} \sum_{s=1}^{\infty} \sum_{\substack{n_{1}+n_{2}+n_{3}=s \\
\mathbf{r}_{1}+\mathbf{r}_{2}+\mathbf{r}_{3}=\mathbf{p}}}\left|\mathbf{r}_{1}\right|\left|\mathbf{r}_{2}\right|\left|\mathbf{r}_{3}\right|\left|\widehat{\boldsymbol{\xi}}_{\mathbf{r}_{1}}^{\left(n_{1}\right)}\right|\left|\widehat{\boldsymbol{\xi}}_{\mathbf{r}_{2}}^{\left(n_{2}\right)}\right|\left|\widehat{\boldsymbol{\xi}}_{\mathbf{r}_{3}}^{\left(n_{3}\right)}\right| t^{s} \\
= & T+\frac{\zeta^{2}(t)}{\sqrt{2}}+\frac{\zeta^{3}(t)}{6},
\end{aligned}
$$

where $T \equiv \Gamma t$ is a dimensionless time; we have used the geometric interpretation of scalar and vector products and applied the inequalities $1 \leq|\cos \theta|+|\sin \theta| \leq \sqrt{2}$ that hold for any angle $\theta$.

The inequality (2.24) is equivalent to

$$
p(\zeta) \equiv \frac{\zeta^{3}}{6}+\frac{\zeta^{2}}{\sqrt{2}}-\zeta+T \geq 0 .
$$

The discriminant of the polynomial $p$ (2.25),

$$
\Delta=-\frac{3}{4} T^{2}-\frac{5}{\sqrt{2}} T+\frac{7}{6},
$$

is positive at small times, and thus $p$ has three real roots $\zeta_{i}$. By Viète's theorem, the product of the roots is negative; since the extrema of the graph of $p$ lie at points of different signs, we infer that $\zeta_{1}<0<\zeta_{2}<\zeta_{3}$. Differentiating in $T$ the equation for the roots of $p$, we find

$$
\frac{\partial \zeta_{i}}{\partial T}=-\left(\left.\frac{\partial p}{\partial \zeta}\right|_{\zeta=\zeta_{i}}\right)^{-1} .
$$

Consequently, on increasing $T$, the $\operatorname{root} \zeta_{2}$ monotonically increases and $\zeta_{3}$ monotonically 


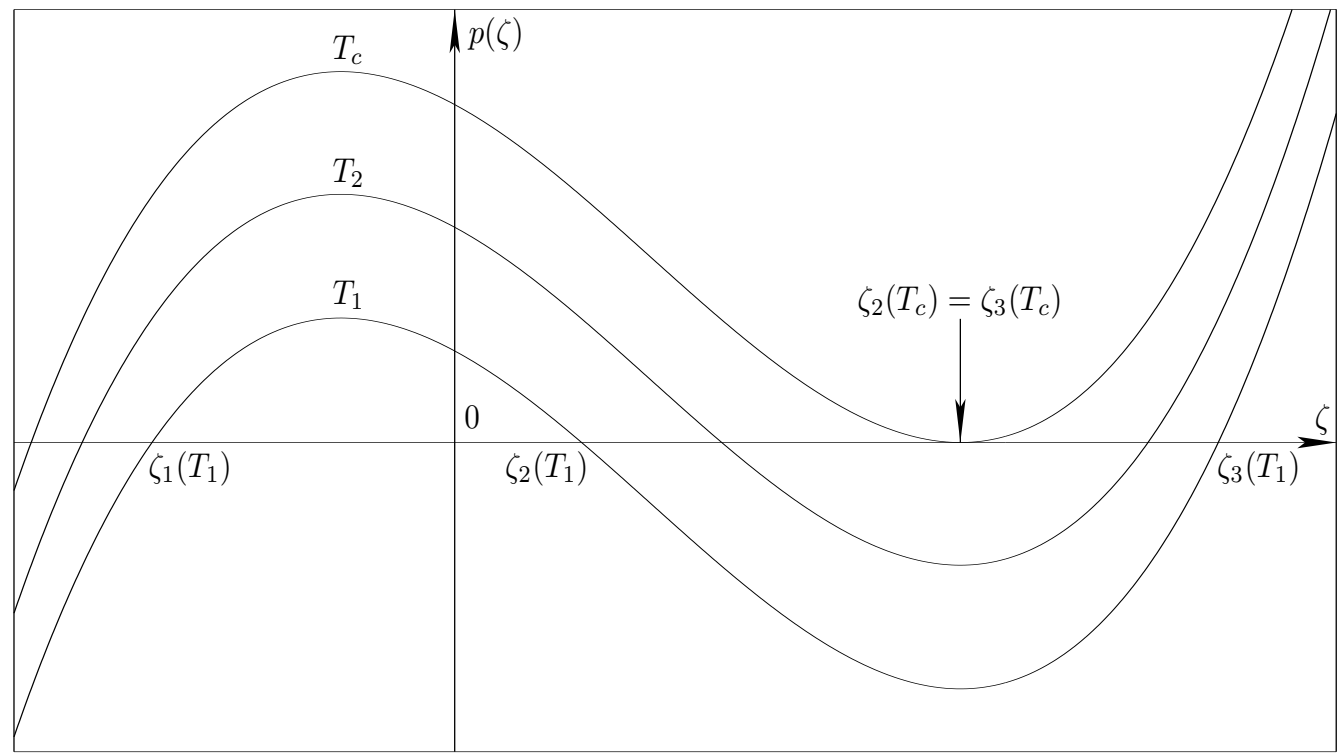

FIGURE 1. A sketch of graphs of the polynomial $p(\zeta)(2.25)$ for three values of $T$, such that $T_{1}<T_{2}<T_{c}$, illustrating the behaviour of real roots of $p$ (the roots are shown as the points of intersection of the graph of $p(\zeta)$ and the horizontal axis). On increasing $T$, the graph slides up as a rigid curve. As a result, the roots $\zeta_{1}$ and $\zeta_{3}$ move to the left (i.e., become smaller), and $\zeta_{2}$ moves to the right (i.e., becomes larger). At the critical value $T_{c}$, for which $\partial p / \partial \zeta=0$ and the discriminant $\Delta$ vanishes, the two roots $\zeta_{2}$ and $\zeta_{3}$ collide and disappear (with emergence of a pair of complex conjugate roots, which cannot be shown in the plot).

decreases till the two roots collide (i.e., $\zeta_{2}=\zeta_{3}$ ) giving birth to a pair of complex roots (see Fig. 1). This occurs at a critical value

$$
T=T_{c}=(8-5 \sqrt{2}) / 3 \approx 0.3096
$$

for which $\Delta=0$. For $T<T_{c}$, inequality (2.25) implies $\zeta \leq \zeta_{2}$. Therefore, the series $\sum_{s, \mathbf{p}}\left|\widehat{\boldsymbol{\xi}}_{\mathbf{p}}^{(s)}(t)\right| t^{s}$ converges for $t<t_{c} \equiv T_{c} / \Gamma$, and hence at any Lagrangian position a the displacement $\boldsymbol{\xi}(\boldsymbol{a}, t)=\sum_{s, \mathbf{p}} \widehat{\boldsymbol{\xi}}_{\mathbf{p}}^{(s)}(t) \mathrm{e}^{\mathbf{i} \mathbf{p} \cdot \boldsymbol{a}} t^{s}$ is analytic in complex time $t$ in the disk $|t|<t_{c}$.

We have just derived the bound $\zeta(t) \leq \zeta_{2}$ under the assumption that the displacement is given by its time-Taylor series. The assumption has enabled us to work with the infinite series, but this is actually not required. A complete mathematical proof proceeds as follows. First, by essentially repeating the arguments of this section, one can show that for $t<t_{c}$ any partial sum of the series (2.23) is also bounded by $\zeta_{2}$. This bound being uniform, we conclude that the series (2.11) for $\boldsymbol{\xi}(\boldsymbol{a}, t)$ converges in the disk $|t|<t_{c}$ for any $\boldsymbol{a}$, and defines an analytic function of time. Consequently, the $s$-th coefficient of the generating function (2.23) is bounded by $\zeta_{2} t_{c}^{-s}$. Second, one substitutes the truncated series (2.11) into equations (2.7) and (2.8). Using our bound for the coefficients in (2.11), we easily find that for $t<t_{c}$ the discrepancies decay as $C S^{2}\left(t / t_{c}\right)^{S}\left(1-t / t_{c}\right)^{-1}$ and $C S^{3}\left(t / t_{c}\right)^{S}\left(1-t / t_{c}\right)^{-1}$, respectively, where $S$ denotes the number of the terms kept and $C$ is a constant. Thus, in the disk of convergence, the formal solution that we have constructed in this section is a classical solution to Cauchy's equations. 


\subsubsection{A normed-space proof}

It may be instructive to give an alternative proof, that is not tailored to the specific functional space to which the initial vorticity belongs, while emphasizing the key ingredients of such a proof.

It is crucial for further developments to reexpress the pair of recurrence relations (2.12)(2.13) as a single recurrence for the (Lagrangian) gradients of the Taylor coefficients (the latter are vectors, so their gradients are 2-tensors). We take the curl of (2.12) and the gradient of (2.13), solve the resultant Poisson equation and finally take one (tensor) gradient to obtain

$$
\begin{aligned}
\nabla_{\mu}^{\mathrm{L}} \xi_{\nu}^{(s)}= & \nabla_{\mu}^{\mathrm{L}} v_{\nu}^{\mathrm{init}} \delta_{1}^{s}+\sum_{\substack{1 \leq j \leq 3, j \neq \nu}} \mathcal{C}_{\mu j}\left(\sum_{\substack{1 \leq k \leq 3 \\
0<m<s}} \frac{2 m-s}{s}\left(\nabla_{\nu}^{\mathrm{L}} \xi_{k}^{(m)}\right) \nabla_{j}^{\mathrm{L}} \xi_{k}^{(s-m)}\right) \\
& +\mathcal{C}_{\mu \nu}\left(\sum_{\substack{1 \leq i<j \leq 3 \\
0<m<s}}\left(\left(\nabla_{j}^{\mathrm{L}} \xi_{i}^{(m)}\right) \nabla_{i}^{\mathrm{L}} \xi_{j}^{(s-m)}-\left(\nabla_{i}^{\mathrm{L}} \xi_{i}^{(m)}\right) \nabla_{j}^{\mathrm{L}} \xi_{j}^{(s-m)}\right)\right. \\
& \left.-\sum_{\substack{i, j, k \\
l+m+n=s}} \varepsilon_{i j k}\left(\nabla_{i}^{\mathrm{L}} \xi_{1}^{(l)}\right)\left(\nabla_{j}^{\mathrm{L}} \xi_{2}^{(m)}\right) \nabla_{k}^{\mathrm{L}} \xi_{3}^{(n)}\right) .
\end{aligned}
$$

Here, $\mathcal{C}_{i j} \equiv \nabla^{-2} \nabla_{i}^{\mathrm{L}} \nabla_{j}^{\mathrm{L}}$ and $\nabla_{i}^{\mathrm{L}} \nabla_{j}^{\mathrm{L}}$ denotes the second-order partial derivative $\partial^{2} / \partial a_{i} \partial a_{j}$. The operator $\nabla^{-2}$, as already stated, is the inverse of the (Lagrangian) Laplacian: given a periodic function $f$ with zero spatial mean over the periodic cell, $\nabla^{-2} f$ is defined as the unique periodic function $\psi$ with zero spatial mean, solving $\nabla^{2} \psi=f$. The structure of (2.29) has an important property: for a given order $s$, the 9-component tensor $\nabla^{\mathrm{L}} \boldsymbol{\xi}^{(s)}$ is expressed in terms of products of gradients of lower-order Taylor coefficients, and of the operators $\mathcal{C}_{i j}$ acting on these products.

Operators such as $\mathcal{C}_{i j} \equiv \nabla^{-2} \nabla_{i}^{\mathrm{L}} \nabla_{j}^{\mathrm{L}}$ have been used in potential theory and applied mathematics since at least the beginning of the 20th century (see Section 2.5). They are instances of what is now sometimes called Calderon-Zygmund operators. In view of the importance of these operators here, we shall call them the "fundamental CalderonZygmund" (fCZ) operators.

Suppose a functional norm has the following two properties: $(i)$ the norms of the fCZ operators are bounded; (ii) the norm of a product is bounded by the product of norms of its factors. Then clearly relation (2.29) enables us to control the norm of the gradient of each subsequent Taylor coefficient. Actually, we have already used, albeit without stressing it, this structural property of the recurrence relations for the gradients of Taylor coefficients in the elementary proof.

We now define a norm which, as we shall show, has the two required properties: For a scalar-valued space-periodic function

$$
f(\boldsymbol{a})=\sum_{\mathbf{p}} \widehat{f}_{\mathbf{p}} \mathrm{e}^{\mathrm{ip} \cdot \boldsymbol{a}}
$$

we set

$$
\|f\| \equiv \sum_{\mathbf{p}}\left|\widehat{f}_{\mathbf{p}}\right|
$$


Following a standard approach, we then set

$$
\|\mathbf{f}\| \equiv \max _{j}\left\|f_{j}\right\|
$$

for a space-periodic vector field $\mathbf{f}$, and

$$
\left\|\nabla^{\mathrm{L}} \mathbf{f}\right\| \equiv \max _{i}\left\|\nabla_{i}^{\mathrm{L}} \mathbf{f}\right\|=\max _{i, j}\left\|\nabla_{i}^{\mathrm{L}} f_{j}\right\|
$$

for the tensor $\nabla^{\mathrm{L}} \mathbf{f}$. Actually, (2.33) is equivalent to the norm $\sum_{\mathbf{p}}|\mathbf{p}|\left|\widehat{\boldsymbol{\xi}}_{\mathbf{p}}^{(s)}\right|$ of $\nabla^{\mathrm{L}} \boldsymbol{\xi}^{(s)}$, used in the elementary proof in the previous section. Other norms having the two required properties will be used in subsequent sections.

$i$. Boundedness of fCZ operators $\mathcal{C}_{i j}$

The operator $\mathcal{C}_{i j}$ is a composition of two derivatives and the inverse Laplacian in Lagrangian variables. For given boundary conditions, applying the inverse Laplacian amounts to solving a Poisson equation. This is a nonlocal operation. Formally counting the number of differentiations involved in the fCZ operator, we see that it is a kind of zeroth-order nonlocal differential operator - in the theory of partial differential operators such an operator is called a pseudodifferential operator of order zero. For the norm $\|\cdot\|$, the fCZ operator $\mathcal{C}_{i j}$ is bounded; actually its norm is unity. Indeed, for any space-periodic function $f$ expanded in a Fourier series (2.30), we have

$$
\left\|\mathcal{C}_{i j} f\right\|=\left\|\sum_{\mathbf{p} \neq 0} \frac{p_{i} p_{j}}{|\mathbf{p}|^{2}} \widehat{f}_{\mathbf{p}} \mathrm{e}^{\mathbf{i} \cdot \boldsymbol{a}}\right\|=\sum_{\mathbf{p} \neq 0} \frac{\left|p_{i} p_{j}\right|}{|\mathbf{p}|^{2}}\left|\widehat{f}_{\mathbf{p}}\right| \leq \sum_{\mathbf{p}}\left|\widehat{f}_{\mathbf{p}}\right|=\|f\|,
$$

as stated.

ii. A bound for the norms of a product

We now show that the norm (2.31) has the second desired property, i.e. the norm of a product is bounded by the product of norms of the factors. Consider two functions $f$ and $g$, whose Fourier coefficients are $\widehat{f}_{\mathbf{p}}$ and $\widehat{g}_{\mathbf{p}}$, respectively. Clearly,

$$
\|f g\|=\sum_{\mathbf{p}}\left|\sum_{\mathbf{n}} \widehat{f}_{\mathbf{n}} \widehat{g}_{\mathbf{p}-\mathbf{n}}\right| \leq \sum_{\mathbf{p}} \sum_{\mathbf{n}}\left|\widehat{f}_{\mathbf{n}}\right|\left|\widehat{g}_{\mathbf{p}}\right|=\|f\|\|g\|,
$$

as required. A linear functional space equipped with a norm with this property is called an algebra.

The right-hand side of (2.29) involves three sums. For a given index of summation $m$, each of the first two sums involve 6 quadratic products of various $\nabla_{i^{\prime}}^{\mathrm{L}} \xi^{\left(j^{\prime}\right)}$; for given $l$ and $m$, the third sum involves 6 cubic products of such components. Using this and applying $i$ and $i i$, we bound the norm of the various terms in the right-hand side of (2.29) and obtain

$$
\begin{aligned}
\left\|\nabla^{\mathrm{L}} \boldsymbol{\xi}^{(s)}\right\| \leq & \left\|\nabla^{\mathrm{L}} \boldsymbol{v}^{(\mathrm{init})}\right\| \delta_{1}^{s}+12 \sum_{m+n=s}\left\|\nabla^{\mathrm{L}} \xi^{(m)}\right\|\left\|\nabla^{\mathrm{L}} \xi^{(n)}\right\| \\
& +6 \sum_{l+m+n=s}\left\|\nabla^{\mathrm{L}} \xi^{(l)}\right\|\left\|\nabla^{\mathrm{L}} \xi^{(m)}\right\|\left\|\nabla^{\mathrm{L}} \xi^{(n)}\right\| .
\end{aligned}
$$

We then introduce the generating function

$$
\widetilde{\zeta}(t) \equiv \sum_{s=1}^{\infty}\left\|\nabla^{\mathrm{L}} \boldsymbol{\xi}^{(s)}\right\| t^{s},
$$

similarly to what we did in the elementary proof. Multiplying (2.36) by $t^{s}$ and summing 
over $s \geq 1$ yields

$$
6 \widetilde{\zeta}^{3}+12 \widetilde{\zeta}^{2}-\widetilde{\zeta}+\left\|\nabla^{\mathrm{L}} \boldsymbol{v}^{(\mathrm{init})}\right\| t \geq 0 .
$$

Inequality (2.38) has the same nature as (2.25), and the remainder of the proof is along the same lines as in the elementary proof. Time analyticity is guaranteed by (2.38) until the critical time $\approx 0.0204 /\left\|\nabla^{\mathrm{L}} \boldsymbol{v}^{\text {(init) }}\right\|$.

Neglecting in the normed-space proof fine details of equations arising in the Fourier space in order to gain a higher generality has resulted in a quantitative (but not qualitative) deterioration of the bounds (for instance, the coefficient in the cubic term in (2.38) is 36 times larger than the one in (2.25)). Although the dimensionless time is defined differently in the "elementary" and the "normed-space" proofs, one easily finds, using the inequality $\left\|\nabla^{\mathrm{L}} \boldsymbol{\xi}^{(s)}\right\| \leq \Gamma \leq 6\left\|\nabla^{\mathrm{L}} \boldsymbol{\xi}^{(s)}\right\|$, that the bound for the radius of convergence of the Taylor series is at least 2.53 times larger in the "elementary" proof.

\subsection{Analytic initial vorticity}

Considering again $2 \pi$-periodic flows, we now assume that the initial condition is an analytic function of the three space variables. We shall prove that the Lagrangian map is a space-time analytic function over the whole periodic domain and until times that again depend only on the initial velocity gradient.

For this, we employ a Gevrey-type norm, that takes advantage of the exponential decrease with wavenumber of the spatial Fourier coefficients of analytic functions. For $\sigma>0$ and a scalar-valued function $f(2.30)$, we define

$$
\|f\|_{\sigma} \equiv \sum_{\mathbf{p}} \mathrm{e}^{\sigma|\mathbf{p}|}\left|\widehat{f}_{\mathbf{p}}\right|
$$

and for a vector field $\mathbf{f}$,

$$
\left\|\nabla^{\mathrm{L}} \mathbf{f}\right\|_{\sigma} \equiv \max _{i, j}\left\|\nabla_{i}^{\mathrm{L}} f_{j}\right\|_{\sigma}
$$

Since the initial velocity $\boldsymbol{v}^{(\mathrm{init})}(\boldsymbol{a})$ is a periodic analytic function, for some $\sigma>0$,

$$
\left\|\nabla^{\mathrm{L}} \boldsymbol{v}^{(\mathrm{init})}\right\|_{\sigma}=\max _{i j} \sum_{\mathbf{p}}\left|p_{i}\right| \mathrm{e}^{\sigma|\mathbf{p}|}\left|\left(\widehat{\boldsymbol{v}}_{\mathbf{p}}\right)_{j}\right|
$$

is finite. This guarantees that the Fourier series for the initial velocity and (2.15) for the initial vorticity converge for complex $\boldsymbol{a}$ in the strip $|\operatorname{Im} z|<\sigma$ around the real domain. Moreover, the supremum of $\sigma$, for which the sum (2.41) converges, can be identified as the distance from the real space $\mathbb{R}^{3}$ to the nearest singularity of the analytic function $\nabla^{\mathrm{L}} \boldsymbol{v}^{\text {(init) }}(\boldsymbol{a})$ in the complex three-dimensional space. As in Section 2.3.2, we consider the generating function

$$
\widetilde{\zeta}_{\sigma}(t) \equiv \sum_{s=1}^{\infty}\left\|\nabla^{\mathrm{L}} \boldsymbol{\xi}^{(s)}\right\|_{\sigma} t^{s}
$$

If we show that the generating function is finite until $t=t_{c}(\sigma)$, spatio-temporal analyticity until that time follows. This will hold if we demonstrate the two required properties of the norm $\|\cdot\|_{\sigma}$. Their proofs are similar mutatis mutandis to those given in the previous subsection in (2.34) and (2.35).

Then, by repeating the normed-space proof almost literally, we derive the inequality

$$
6 \widetilde{\zeta}_{\sigma}^{3}+12 \widetilde{\zeta}_{\sigma}^{2}-\widetilde{\zeta}_{\sigma}+t\left\|\nabla^{\mathrm{L}} \boldsymbol{v}^{(\mathrm{init})}\right\|_{\sigma} \geq 0,
$$

from which analyticity in space and time follows.

More careful estimations, as in the elementary proof of Section 2.3.1 show that the 
generating function

$$
\zeta_{\sigma}(t) \equiv \sum_{s=1}^{\infty}\left(\sum_{\mathbf{p}}|\mathbf{p}| \mathrm{e}^{\sigma|\mathbf{p}|}\left|\widehat{\boldsymbol{\xi}}_{\mathbf{p}}^{(s)}\right|\right) t^{s}
$$

satisfies the inequality

$$
\frac{\zeta_{\sigma}^{3}}{6}+\frac{\zeta_{\sigma}^{2}}{\sqrt{2}}-\zeta_{\sigma}+t \Gamma_{\sigma} \geq 0
$$

where $\Gamma_{\sigma} \equiv\left\|\boldsymbol{\omega}^{(\mathrm{init})}\right\|_{\sigma}$. Reasoning precisely as in Section 2.3.1, we infer that $\zeta_{\sigma}$ remains bounded and therefore the Lagrangian map is analytic for $t<T_{c} / \Gamma_{\sigma}$ where $T_{c} \approx 0.3096$, as given by (2.28), and its singularities, if any, are at a distance at least $\sigma$ from the real space. Letting $\sigma \searrow 0$, and observing that $\Gamma_{\sigma}$ is an increasing continuous function of $\sigma$, we conclude that $\boldsymbol{\xi}(\boldsymbol{a}, t)$ is guaranteed to possess spatio-temporal analyticity for $t<t_{c} \equiv T_{c} / \Gamma_{0}$.

For analytic initial data, analyticity of the Lagrangian map in space and time is not surprising, given that in Eulerian coordinates it has been known for a long time that, with analytic initial data, the solution is analytic in space and time (Benachour 1976; spatial analyticity of space-periodic solutions was further considered by Levermore \& Oliver 1997, Kukavica \& Vicol 2009 and Zheligovsky 2011). The Lagrangian map is the solution to $\dot{\boldsymbol{x}}=\boldsymbol{v}(\boldsymbol{x}, t)$ with $\boldsymbol{x}(0)=\boldsymbol{a}$, where $\boldsymbol{v}(\boldsymbol{x}, t)$ is the Eulerian velocity. From this one can in principle establish analyticity of the Lagrangian map, using the theory of ODEs of complex variables.

Nevertheless, with our derivation, we have gained something essential: the latter proof, by way of Eulerian analyticity, will not work beyond complex times whose moduli exceed the radius of convergence of the Taylor series in $t$ for any Eulerian $\boldsymbol{x}$. This Eulerian radius of convergence does not depend only on the initial velocity gradient, as is the case of the time of guaranteed analyticity by our Cauchy-formulation-based technique, but also on the initial velocity itself. This can be seen by adding to the initial flow a large uniform velocity $\boldsymbol{U}$. As a consequence, a singularity at a given complex space location $\boldsymbol{x}_{\star}$ within a distance $\delta$ of the real domain will be swept past a fixed (Eulerian) observer at speed $\boldsymbol{U}$ and, to leading order, it will also be seen as a complex singularity in time at a location whose imaginary part is $\delta /|\boldsymbol{U}|$. Thus as $|\boldsymbol{U}|$ is increased, the Eulerian radius of convergence decreases. Note that the argument is basically equivalent to the use of the Taylor hypothesis, discussed in the Introduction.

This argument has an important implication for numerical simulation of ideal incompressible flow, particularly when high precision is needed, e.g. to study the appearance of possible singularities. The simplest way to achieve high precision in temporal schemes is to use a high-order scheme, for example a high-order truncated Taylor series in time, the time-step of which cannot normally exceed the radius of convergence of the Taylor series. In Eulerian coordinates this may result in a much smaller permissible time step than in Lagrangian coordinates. We shall discuss this issue elsewhere.

Finally, let us remark that the use of Lagrangian coordinates has proved useful for establishing the spatial analyticity of solutions in a 3D fluid region with a boundary, and deriving bounds for the rate of decay of their radius of spatial analyticity (Kukavica \& Vicol 2011).

\subsection{Hölder-continuous initial vorticity}

Again, we here assume spatial periodicity and denote the elementary periodicity cell by $\mathbb{T}^{3} \equiv[0,2 \pi]^{3}$. We assume that the initial vorticity is Hölder-continuous with some 
exponent $0<\alpha<1$. This means that its Hölder norm

$$
\left|\boldsymbol{\omega}^{(\mathrm{init})}\right|_{\alpha} \equiv \max _{\boldsymbol{a} \in \mathbb{T}^{3}}\left|\boldsymbol{\omega}^{(\mathrm{init})}(\boldsymbol{a})\right|+\sup _{\boldsymbol{a}_{1}, \boldsymbol{a}_{2} \in \mathbb{T}^{3}, \boldsymbol{a}_{1} \neq \boldsymbol{a}_{2}} \frac{\left|\boldsymbol{\omega}^{(\mathrm{init})}\left(\boldsymbol{a}_{1}\right)-\boldsymbol{\omega}^{(\mathrm{init})}\left(\boldsymbol{a}_{2}\right)\right|}{\left|\boldsymbol{a}_{1}-\boldsymbol{a}_{2}\right|^{\alpha}}
$$

is finite. Such a function is continuous but may be non-differentiable, and its increments between two neighbouring points $\boldsymbol{a}_{1}$ and $\boldsymbol{a}_{2}$ may behave as $\left|\boldsymbol{a}_{1}-\boldsymbol{a}_{2}\right|^{\alpha}$.

For such flows we give a proof of the finite-time temporal analyticity of the Lagrangian map. It is similar to the proof by Frisch \& Zheligovsky (2014), but builds on the material of Section 2.3.2 and is more detailed, in order to have a self-contained presentation for fluid dynamicists.

i. Boundedness of the $f C Z$ operators $\mathcal{C}_{i j}$

Here we consider the following statement: Let a function $f(\boldsymbol{a})$ be periodic and Höldercontinuous of exponent $0<\alpha<1$, then

$$
\left|\nabla^{-2} \nabla_{i}^{\mathrm{L}} \nabla_{j}^{\mathrm{L}} f(\boldsymbol{a})\right|_{\alpha} \leq \Theta_{\alpha}|f|_{\alpha},
$$

where $\Theta_{\alpha}$ does not depend on $f$ and is bounded by $C / \alpha$. In the language of electrostatics, an equivalent statement would be that if the charge density is Hölder-continuous, so is the gradient of the electric field. In hydrodynamics, an analogous result is that, if the vorticity is Hölder-continuous, so is the gradient of the velocity, the latter being given by a Biot-Savart integral. Note that a vorticity that is merely continuous can give rise to an arbitrarily large velocity gradient. Hence such a vorticity could be shear-stretched arbitrarily fast, leading to a vanishing interval of assured regularity.

Closely related results have been proved by mathematicians in the early twentieth century. Lichtenstein (1925) stated a similar result in the entire space $\mathbb{R}^{3}$, giving a reference to Korn (1907) and mentioning that "it does not present serious difficulties but requires, as is known, a series of rather cumbersome thoughts." He used it to prove various results regarding the initial-value problem for the 3D incompressible Euler equations, including its well-posedeness for a finite time when the initial vorticity is Hölder-continuous (Lichtenstein 1927). Chemin (1992) used the boundedness of the fCZ operators (essentially, in the Eulerian framework) to prove that when the initial vorticity is Hölder-continuous, the fluid particle trajectories are infinitely differentiable in time.

There exist two types of proofs of the boundedness of the $\mathrm{fCZ}$ operators similar to (2.47). When the problem is set in the entire $\mathbb{R}^{3}$ space, the Green function for the Poisson equation is basically the Coulomb potential $1 / r$ and proofs can be given by bounding suitable singular integral operators in physical space. With periodicity conditions, the problem is somewhat more cumbersome, although the Green function still behaves as $1 / r$ at distances $r$ small compared to the period. The method, using so-called Poisson integrals, (see, e.g., Stein 1970, Chapters 3 and 4) can handle both instances; it is rather elementary, but definitely too long for presenting it here, even in a summarised form. To give the reader an idea of why the boundedness holds, we shall discuss the case of the entire space and for simplicity assume that the given function $f$ is not only Höldercontinuous of exponent $\alpha$, but also vanishes outside a bounded domain (i.e., is compactly supported). We here follow Gilbarg \& Trudinger (1998, Chapter 4) and Majda \& Bertozzi (2002, Chapter 4).

The solution to the three-dimensional Poisson equation

$$
\nabla^{2} \phi=f
$$

is a convolution

$$
\phi(\boldsymbol{a})=\int f(\boldsymbol{y}) G(\boldsymbol{a}-\boldsymbol{y}) \mathrm{d} \boldsymbol{y}
$$


where the integral is taken over the whole space and the Green function $G(\boldsymbol{a})$ is

$$
G(\boldsymbol{a})=-\frac{1}{4 \pi|\boldsymbol{a}|} .
$$

In spite of the singular behaviour of the Green function at $\boldsymbol{a}=0$, the integral (2.49) converges. Furthermore, we can differentiate it once with respect to $\boldsymbol{a}$ by just differentiating the Green function to obtain

$$
\nabla_{i}^{\mathrm{L}} \phi(\boldsymbol{a})=\int f(\boldsymbol{y}) \nabla_{i}^{\mathrm{L}} G(\boldsymbol{a}-\boldsymbol{y}) \mathrm{d} \boldsymbol{y} .
$$

If however we wish to differentiate twice, we cannot proceed in the same way, since the second derivatives of the Green function have a strong, $|\boldsymbol{a}|^{-3}$, singularity which, in three dimensions, could lead to a logarithmic divergence at short distance. This is overcome in the following way. In principle to differentiate a convolution integral such as (2.51), we can differentiate either of the factors. So, we can apply one of the differentiations to $f$ rather than to the Green function. For this we need to assume temporarily that $f$ is differentiable. As we shall see, the final expression for the second derivative of $\phi$ does not involve derivatives of $f$ and holds for functions which are merely Hölder-continuous. Standard arguments allow us then to justify the formula for such functions. Thus,

$$
\nabla_{i}^{\mathrm{L}} \nabla_{j}^{\mathrm{L}} \phi(\boldsymbol{a})=\int \frac{\partial}{\partial a_{j}} f\left(\boldsymbol{a}-\boldsymbol{y}^{\prime}\right) \frac{\partial}{\partial y_{i}^{\prime}} G\left(\boldsymbol{y}^{\prime}\right) \mathrm{d} \boldsymbol{y}^{\prime},
$$

where we have changed variables from $\boldsymbol{y}$ to $\boldsymbol{y}^{\prime} \equiv \boldsymbol{a}-\boldsymbol{y}$. We note that

$$
\frac{\partial}{\partial a_{j}} f\left(\boldsymbol{a}-\boldsymbol{y}^{\prime}\right)=-\frac{\partial}{\partial y_{j}^{\prime}} f\left(\boldsymbol{a}-\boldsymbol{y}^{\prime}\right)
$$

and subtract a counterterm, which is clearly zero, from (2.52) to get

$$
\nabla_{i}^{\mathrm{L}} \nabla_{j}^{\mathrm{L}} \phi(\boldsymbol{a})=-\int \frac{\partial}{\partial y_{j}^{\prime}}\left(f\left(\boldsymbol{a}-\boldsymbol{y}^{\prime}\right)-f(\boldsymbol{a})\right) \frac{\partial}{\partial y_{i}^{\prime}} G\left(\boldsymbol{y}^{\prime}\right) \mathrm{d} \boldsymbol{y}^{\prime} .
$$

Now, we return to the original spatial variable $\boldsymbol{y}$ and observe that, since $f$ was assumed to vanish outside of a bounded set, called its support, we do not have to integrate over the whole space. It is convenient to chose a set $\Omega$ with a smooth boundary, for example a sphere, whose boundary $\partial \Omega$ is at a strictly positive distance from the support of $f$. Using the Gauss-Ostrogradsky divergence theorem, we obtain

$$
\nabla_{i}^{\mathrm{L}} \nabla_{j}^{\mathrm{L}} \phi=\int_{\Omega}(f(\boldsymbol{y})-f(\boldsymbol{a})) \nabla_{i}^{\mathrm{L}} \nabla_{j}^{\mathrm{L}} G(\boldsymbol{a}-\boldsymbol{y}) \mathrm{d} \boldsymbol{y}-f(\boldsymbol{a}) \int_{\partial \Omega} \nabla_{i}^{\mathrm{L}} G(\boldsymbol{a}-\boldsymbol{y}) \nu_{j}(\boldsymbol{y}) \mathrm{d} \sigma(\boldsymbol{y}),
$$

where $\boldsymbol{\nu}$ is the outward unit vector normal to $\partial \Omega$ (note that $f(\boldsymbol{y})=0$ for $\boldsymbol{y}$ on $\partial \Omega$ ).

The first integral in the right-hand side is nearly what we would have obtained by illegitimate naive double differentiation of the Green function. However, $f$ appears now only through its increments. By Hölder continuity, these are bounded by $|f|_{\alpha}|\boldsymbol{y}-\boldsymbol{a}|^{\alpha}$, thus preventing the aforementioned logarithmic divergence. Specifically, we have

$$
\left|\int_{\Omega}(f(\boldsymbol{y})-f(\boldsymbol{a})) \nabla_{i}^{\mathrm{L}} \nabla_{j}^{\mathrm{L}} G(\boldsymbol{a}-\boldsymbol{y}) \mathrm{d} \boldsymbol{y}\right| \leq c|f|_{\alpha} \int_{\Omega}|\boldsymbol{y}-\boldsymbol{a}|^{\alpha-3} \mathrm{~d} \boldsymbol{y} \leq \frac{C}{\alpha}|f|_{\alpha},
$$

where $c$ and $C$ are suitable positive constants. To bound the Hölder norm of the first integral in (2.55), we also need to bound its increments. Establishing such bounds is a bit technical but quite straightforward (see, e.g., Gilbarg \& Trudinger 1998, Chapter 4).

As to the second, surface integral in $(2.55)$ - since the boundary $\partial \Omega$ is at a positive 
distance from the set where $f \neq 0$, this integral is an indefinitely differentiable function on the support of $f$. We have thus established the inequality (2.47), albeit in the framework of an entire-space formulation of the Poisson problem with a right-hand side that has bounded support.

ii. Bounds for products of Hölder-continuous functions

The other property we need is straightforward. Let $f$ and $g$ be Hölder-continuous functions of exponents $\alpha$. Then

$$
\begin{aligned}
|f g|_{\alpha} \leq & \max _{\boldsymbol{a} \in \mathbb{T}^{3}}|f(\boldsymbol{a})| \max _{\boldsymbol{a} \in \mathbb{T}^{3}}|g(\boldsymbol{a})|+\max _{\boldsymbol{a}_{1} \in \mathbb{T}^{3}}\left|g\left(\boldsymbol{a}_{1}\right)\right| \sup _{\boldsymbol{a}_{1}, \boldsymbol{a}_{2} \in \mathbb{T}^{3}, \boldsymbol{a}_{1} \neq \boldsymbol{a}_{2}} \frac{\left|f\left(\boldsymbol{a}_{1}\right)-f\left(\boldsymbol{a}_{2}\right)\right|}{\left|\boldsymbol{a}_{1}-\boldsymbol{a}_{2}\right|} \\
& +\max _{\boldsymbol{a}_{2} \in \mathbb{T}^{3}}\left|f\left(\boldsymbol{a}_{2}\right)\right| \sup _{\boldsymbol{a}_{1}, \boldsymbol{a}_{2} \in \mathbb{T}^{3}, \boldsymbol{a}_{1} \neq \boldsymbol{a}_{2}} \frac{\left|g\left(\boldsymbol{a}_{1}\right)-g\left(\boldsymbol{a}_{2}\right)\right|}{\left|\boldsymbol{a}_{1}-\boldsymbol{a}_{2}\right|} \leq|f|_{\alpha}|g|_{\alpha},
\end{aligned}
$$

as required.

Having established the two key properties of the Hölder norm, we can now prove analyticity along the same lines as before: we introduce the generating function

$$
\zeta_{\alpha}(t) \equiv \sum_{s=1}^{\infty}\left|\nabla^{\mathrm{L}} \boldsymbol{\xi}^{(s)}\right|_{\alpha} t^{s}
$$

where we use the notation $\left|\nabla^{\mathrm{L}} \mathbf{f}\right|_{\alpha} \equiv \max _{i, j}\left|\nabla_{i}^{\mathrm{L}} f_{j}\right|_{\alpha}$. Applying (2.47) and (2.57) to (2.29), we deduce the inequality

$$
6 \Theta_{\alpha} \zeta_{\alpha}^{3}+12 \Theta_{\alpha} \zeta_{\alpha}^{2}-\zeta_{\alpha}+T \geq 0
$$

where $T=t\left|\nabla^{\mathrm{L}} \boldsymbol{v}^{\text {(init) }}\right|_{\alpha}$. Like in Section 2.3.1, this implies analyticity of the Lagrangian map until a time inversely proportional to the Hölder norm of the initial vorticity.

It is well-known that absolute summability of the Fourier series and Hölder regularity define distinct classes of functions, although the difference is subtle. For example, if a function is Hölder-continuous of exponent $\alpha>1 / 2$, a theorem of Bernstein implies the absolute convergence of the Fourier series (see, e.g., Katznelson 2004, Section 6.1). This result is sharp: for $\alpha=1 / 2$, there exist functions that are Hölder-continuous without having an absolutely summable Fourier series; a classical example is the HardyLittlewood series $\sum_{n \geq 1} n^{-1} \mathrm{e}^{\mathrm{i} C n \ln n} \mathrm{e}^{\mathrm{i} n q}$ that converges uniformly in the interval $[0,2 \pi]$ to a Hölder-continuous function of exponent $1 / 2$ without being absolutely summable (see, e.g., Zygmund 2002, p. 197).

\subsection{Bounds on the radius of convergence of the time-Taylor series}

After we have established the analyticity in time of the Lagrangian trajectories under various assumptions on the initial conditions, it is natural to ask: what is the radius of convergence of the time-Taylor series that we have constructed? This radius of convergence tells us how far we can evaluate the solution without having to perform analytic continuation. This is important when investigating the issue of finite-time blow up for solutions of the incompressible Euler equations. For example, Brachet et al. (1983) investigated the blow up in the Taylor-Green flow (Taylor \& Green 1937) and showed by analysing the Taylor series for the enstrophy (the space integral of the squared vorticity) that its radius of convergence is determined by a pair of pure imaginary singularities at the complex time locations $t_{\star} \approx \pm 2.18 \mathrm{i}$. Any putative real singularity has to be beyond the disk of convergence, but in practice it is very difficult to analytically continue outside the disk of convergence.

We have by now derived lower bounds for the radius of convergence of the time-Taylor series of the Lagrangian map. Can we obtain also upper bounds? For example, can we 
ascertain that the Lagrangian map is not an entire function with infinite radius of convergence? In general, we have not succeeded in doing this. However, we have constructed analytically the full Lagrangian map and found the radius of convergence to be finite for a steady-state solution to the Euler equations for incompressible fluid. As any Beltrami flow, the well-known ABC flows (see Dombre et al. 1986, and references therein) are steady-state solution to the 3D Euler equations. In general, they have a non-trivial Lagrangian structure, and the ordinary differential equations (ODEs) for the Lagrangian trajectories are not analytically integrable unless one of the coefficients $A, B$ or $C$ vanishes (see Appendix A of Dombre et al. (1986) and Pauls \& Matsumoto (2005)). Here, we consider the symmetric "AB flow"

$$
\boldsymbol{v}\left(x_{1}, x_{2}\right)=\left(-\sin x_{1} \sin x_{2},-\cos x_{1} \cos x_{2}, \sqrt{2} \sin x_{1} \cos x_{2}\right) .
$$

It is easily checked that its vorticity is $\boldsymbol{\omega}\left(x_{1}, x_{2}\right)=\sqrt{2} \boldsymbol{v}\left(x_{1}, x_{2}\right)$ and that it becomes an ABC flow with $C=0$ and $A=B=1$ after a rotation by $\pi / 4$ about the $x_{3}$ axis, a stretching of the coordinates by a factor $\sqrt{2}$ and a suitable permutation of the coordinates. In Section 2.6.1, we calculate the radius of convergence of its Lagrangian map.

The next step (Section 2.6.2) is to improve the lower bounds on the radius of convergence. While the numbers obtained in Section 2.3.1 can be improved by various methods, particularly in the $2 \mathrm{D}$ case, as we shall see, a substantial gap remains between the improved estimates and the actual values for the $\mathrm{AB}$ flow (2.60).

\subsubsection{AB flow: a flow with integrable Lagrangian trajectories}

The AB flow (2.60) is independent of the vertical, $x_{3}$, coordinate. As a consequence, the vertical component of the velocity behaves just as a scalar advected by the horizontal components. The stream function of the horizontal part of the flow is

$$
\psi\left(x_{1}, x_{2}\right)=\sin x_{1} \cos x_{2},
$$

which, like the flow itself, is time-independent in Eulerian coordinates. The Lagrangian map is as usual the solution to $\dot{\boldsymbol{x}}=\boldsymbol{v}$ with the initial condition $\boldsymbol{x}(0)=\boldsymbol{a}$. It is easily checked that the stream function is a Lagrangian invariant. Consequently, the third component of the Lagrangian is just a linear function of time and cannot induce loss of analyticity. Henceforth we consider only the horizontal part of the fluid particle motion, governed by

$$
\dot{x}_{1}=-\sin x_{1} \sin x_{2}, \quad \dot{x}_{2}=-\cos x_{1} \cos x_{2} .
$$

To solve (2.62) we make use of the invariance of the stream function (2.61). Introducing the new dependent variable $w \equiv \sin ^{2} x_{1}$, and assuming without loss of generality that $\sin x_{1} \sin x_{2} \geq 0$, we reduce (2.62) to a separable ODE

$$
\dot{w}=-2 \sqrt{w(1-w)\left(w-\psi^{2}\right)} .
$$

Thus, the solution can be represented in terms of the elliptic integral:

$$
\int_{w(0)}^{w(t)} \frac{\mathrm{d} w}{\sqrt{w(1-w)\left(w-\psi^{2}\right)}}=-2 t .
$$

For our purposes, we do not need much of the theory of elliptic functions. The important point is that the only singularities of the elliptic function $w(t)$ are double poles (see, e.g., Akhiezer 1990). When $t$ approaches a time of singularity $t_{\star}$, the dependent variable $w$ tends to infinity and hence the original variable $x_{1}$ tends to complex infinity. To calculate such singular times we should evaluate the definite integral (2.64) from the real value $w(0)$ (between $\psi^{2}$ and 1) to complex infinity. Consider the contour shown in Fig. 2, 


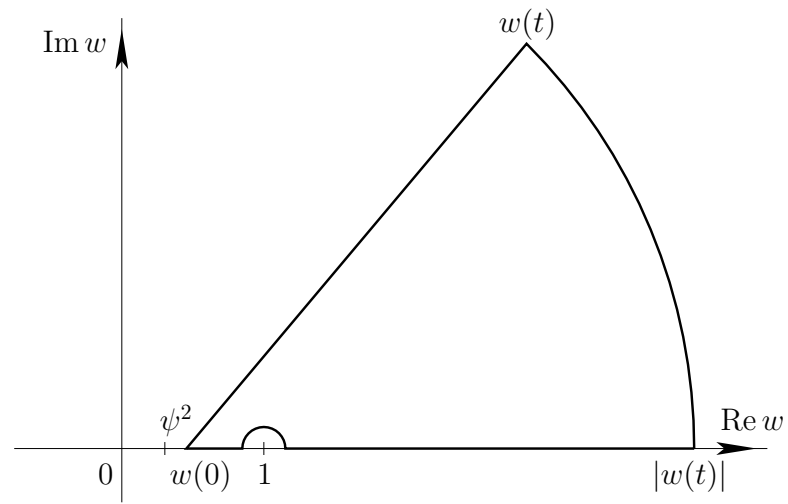

Figure 2. A sketch of the path of integration in the $\mathbb{C}$ plane for the integral (2.64) for computation of the minimal (in absolute value) time of formation of singularity in the AB flow.

designed to avoid inside it singularities of the integrand in (2.64). It is easily shown that the integral over the arc from $w(t)$ to $|w(t)|$ tends to zero as $w(t)$ tends to complex infinity. Letting the radius of the semi-circle around $w=1$ tend to zero, we can replace the integration from $w(0)$ to complex infinity by an integration over the real axis from $w(0)$ to $+\infty$ :

$$
2 t_{\star}=\int_{w(0)}^{1} \frac{\mathrm{d} w}{\sqrt{w(1-w)\left(w-\psi^{2}\right)}}+\mathrm{i} \int_{1}^{\infty} \frac{\mathrm{d} w}{\sqrt{w(w-1)\left(w-\psi^{2}\right)}} .
$$

Next, we want to find the values $\psi$ and $w(0)$ that minimize $\left|t_{\star}\right|$. By (2.65), the imaginary part of $t_{\star}$ does not depend on $w(0)$. Hence the smallest module, for a given $\psi$, is obtained for the smallest real part, namely, for $w(0)=1$. Finally, we have to minimize over $\psi$. Inspection of the second, pure imaginary, integral in (2.65) shows that the minimum is achieved for $\psi=0$. For these parameter values the integral becomes elementary:

$$
t_{\star}=\frac{\mathrm{i}}{2} \int_{1}^{\infty} \frac{\mathrm{d} w}{w \sqrt{w-1}}=\frac{\mathrm{i} \pi}{2}
$$

(it can be evaluated by substituting $w=1+\tan ^{2} \theta$ for $0 \leq \theta \leq \pi / 2$ ). Hence the radius of convergence of the time-Taylor series for the $\mathrm{AB}$ flow is the finite number $\pi / 2$.

\subsubsection{Improvements of bounds for the radius of convergence of the Taylor series}

We shall now show that the lower bound for the radius of convergence of the timeTaylor series for the Lagrangian map can be somewhat improved. For this we shall use the Fourier series formulation and notation of Section 2.3.1. In that section, bounds on the Fourier coefficients of the various time-Taylor coefficients were obtained from the recurrence relations $(2.12)-(2.13)$ and then used to bound the generating function $\zeta$ (2.23). Actually, the first few time-Taylor coefficients can be calculated explicitly from (2.12)-(2.13) and then used to obtain tighter bounds.

For instance, for $s=1$ and $s=2$, we obtain

$$
\widehat{\boldsymbol{\xi}}_{\mathbf{p}}^{(1)}=\frac{\mathbf{i p} \times \widehat{\boldsymbol{\omega}}_{\mathbf{p}}}{|\mathbf{p}|^{2}} ; \quad \widehat{\boldsymbol{\xi}}_{\mathbf{p}}^{(2)}=-\frac{\mathbf{i p}}{2|\mathbf{p}|^{2}} \sum_{0 \neq \mathbf{r} \neq \mathbf{p}} \frac{\left[\mathbf{r}, \mathbf{p}-\mathbf{r}, \widehat{\boldsymbol{\omega}}_{\mathbf{r}}\right]\left[\mathbf{r}, \mathbf{p}-\mathbf{r}, \widehat{\boldsymbol{\omega}}_{\mathbf{p}-\mathbf{r}}\right]}{|\mathbf{r}|^{2}|\mathbf{p}-\mathbf{r}|^{2}} .
$$

Here, the Fourier coefficients of the initial vorticity are denoted, as before, $\widehat{\omega}_{\mathbf{p}}$. Therefore, the bounds (2.21)-(2.22) for the transverse and longitudinal components, and (2.67) yield 
the following bound for the generating function:

$$
\begin{aligned}
\zeta \leq & t \sum_{\mathbf{p}}|\mathbf{p}|\left|\widehat{\boldsymbol{\xi}}_{\mathbf{p}}^{(1)}\right|+t^{2} \sum_{\mathbf{p}}|\mathbf{p}|\left|\widehat{\boldsymbol{\xi}}_{\mathbf{p}}^{(2)}\right|+\sum_{s=3}^{\infty} \sum_{\mathbf{p}}\left(\left|\mathbf{p} \times \widehat{\boldsymbol{\xi}}_{\mathbf{p}}^{(s)}\right|+\left|\mathbf{p} \cdot \widehat{\boldsymbol{\xi}}_{\mathbf{p}}^{(s)}\right|\right) t^{s} \\
\leq & T+\frac{t^{2}}{2} \sum_{\mathbf{p} \neq 0}\left|\sum_{0 \neq \mathbf{r} \neq \mathbf{p}} \frac{\left[\mathbf{r}, \mathbf{p}-\mathbf{r}, \widehat{\boldsymbol{\omega}}_{\mathbf{r}}\right]\left[\mathbf{r}, \mathbf{p}-\mathbf{r}, \widehat{\boldsymbol{\omega}}_{\mathbf{p}-\mathbf{r}}\right]}{|\mathbf{r}|^{2}|\mathbf{p}-\mathbf{r}|^{2}}\right| \\
& +\frac{1}{\sqrt{2}} \sum_{s=3}^{\infty} \sum_{0<m<s, \mathbf{r}}|\mathbf{r}||\mathbf{p}-\mathbf{r}|\left|\widehat{\boldsymbol{\xi}}_{\mathbf{r}}^{(m)}\right|\left|\widehat{\boldsymbol{\xi}}_{\mathbf{p}-\mathbf{r}}^{(s-m)}\right| t^{s} \\
& +\frac{1}{6} \sum_{s=3}^{\infty} \sum_{n_{1}+n_{2}+n_{3}=s}\left|\mathbf{r}_{1}\right|\left|\mathbf{r}_{2}\right|\left|\mathbf{r}_{3}\right|\left|\widehat{\boldsymbol{\xi}}_{\mathbf{r}_{1}}^{\left(n_{1}\right)}\right|\left|\widehat{\boldsymbol{\xi}}_{\mathbf{r}_{2}}^{\left(n_{2}\right)}\right|\left|\widehat{\boldsymbol{\xi}}_{\mathbf{r}_{3}}^{\left(n_{3}\right)}\right| t^{s} \\
= & T+\frac{t^{2}}{2} \sum_{\mathbf{p} \neq 0}\left|\sum_{0 \neq \mathbf{r} \neq \mathbf{p}} \frac{\left[\mathbf{r}, \mathbf{p}-\mathbf{r}, \widehat{\boldsymbol{\omega}}_{\mathbf{r}}\right]\left[\mathbf{r}, \mathbf{p}-\mathbf{r}, \widehat{\boldsymbol{\omega}}_{\mathbf{p}-\mathbf{r}}\right]}{|\mathbf{r}|^{2}|\mathbf{p}-\mathbf{r}|^{2}}\right|+\frac{\zeta^{2}-T^{2}}{\sqrt{2}}+\frac{\zeta^{3}}{6} \\
\leq & T+T^{2}\left(\frac{1}{2}-\frac{1}{\sqrt{2}}\right)+\frac{\zeta^{2}}{\sqrt{2}}+\frac{\zeta^{3}}{6} .
\end{aligned}
$$

Here, $T \equiv t \Gamma=t \sum_{\mathbf{p}}\left|\widehat{\boldsymbol{\omega}}_{\mathbf{p}}\right|$, as before. Observe that (2.69) differs from (2.24) only by a new quadratic term in the dimensionless time $T$. It immediately follows that convergence of the series (2.11) is guaranteed for $t<\widetilde{t}_{c} \equiv \widetilde{T}_{c} / \Gamma$, where the critical dimensionless time $\widetilde{T}_{c}$ satisfies the equation $\widetilde{T}_{c}+(1 / 2-1 / \sqrt{2}) \widetilde{T}_{c}^{2}=T_{c}$ implying

$$
\widetilde{T}_{c}=1+\sqrt{2}-\sqrt{13 / 3} \approx 0.3325 .
$$

Comparison with (2.28) show a modest improvement of about $7 \%$.

So far we were assuming an arbitrary 3D initial condition (within the class of vorticities with summable Fourier coefficients). A more significant improvement can be achieved by constructing bounds for a specific flow in two dimensions. For instance, for the AB flow (2.61), for which we know the exact radius of convergence, the Taylor coefficients of order one and two are easily shown to be

$$
\boldsymbol{\xi}^{(1)}=\left(-\sin a_{1} \sin a_{2},-\cos a_{1} \cos a_{2}\right), \quad \boldsymbol{\xi}^{(2)}=\frac{1}{4}\left(\sin 2 a_{1},-\sin 2 a_{2}\right) .
$$

Consequently, for the $\mathrm{AB}$ flow,

$$
\Gamma=\sum_{\mathbf{p}}|\mathbf{p}|\left|\widehat{\boldsymbol{\xi}}_{\mathbf{p}}^{(1)}\right|=2, \quad \sum_{\mathbf{p}}|\mathbf{p}|\left|\widehat{\boldsymbol{\xi}}_{\mathbf{p}}^{(2)}\right|=1,
$$

and thus the analogue of (2.68) takes the form

$$
T+T^{2}\left(\frac{1}{4}-\frac{1}{\sqrt{2}}\right)-\zeta+\frac{\zeta^{2}}{\sqrt{2}} \geq 0
$$

(in two dimensions the cubic term is absent in (2.68)). In the dimensionless time $T$, analyticity holds now until

$$
T_{\mathrm{AB}}=\frac{2-\sqrt[4]{2}}{\sqrt{8}-1} \approx 0.4434
$$

an improvement of about $43 \%$ over the value given by (2.28). The actual value of the radius of convergence for the $\mathrm{AB}$ flow in dimensionless form is $\pi$, which is still about a factor 7 larger than our improved bound. There is definitely room for further improvement. 


\section{Ideal compressible fluid flow in an Einstein-de Sitter universe}

The simplest model for the dynamics of compressible ideal fluid in the potential case, in any dimension, is the Burgers equation:

$$
\partial_{t} \boldsymbol{v}+(\boldsymbol{v} \cdot \nabla) \boldsymbol{v}=0 ; \quad \boldsymbol{v}=-\nabla \varphi
$$

Its Lagrangian formulation is simply $\ddot{\boldsymbol{x}}=0$, which integrates to $\boldsymbol{x}(\boldsymbol{a}, t)=\boldsymbol{a}+t \boldsymbol{v}^{(\mathrm{init})}(\boldsymbol{a})$. This Lagrangian map is obviously analytic, and even entire, in the time variable. Singularities appear nevertheless, but only when reverting to Eulerian coordinates, because the Lagrangian map ceases to be invertible when its Jacobian $J \equiv \operatorname{det}\left(I+t \nabla^{\mathrm{L}} \boldsymbol{v}^{(\mathrm{init})}\right)$ vanishes for the first time (here $I$ denotes the identity matrix).

There are models of compressible dynamics with far less trivial Lagrangian maps that can be viewed as extensions of the ideal potential Burgers dynamics. Of particular interest are the Euler-Poisson equations, briefly presented in Section 3.1.1, which arise in cosmology and govern the essentially collisionless and thus ideal motion of dark matter. Actually, our interest in analyticity of Lagrangian trajectories in hydrodynamics was triggered by work on the Lagrangian perturbation theory in cosmology (Moutarde et al. 1991; Bouchet et al. 1992, 1995; Buchert 1992, see also Buchert (1989)).

\subsection{The Euler-Poisson equations}

\subsubsection{Basic equations in Eulerian coordinates}

The cosmological Euler-Poisson system of equations takes its shortest form in the so-called comoving coordinates $\boldsymbol{x}$ (moving with the Hubble expansion). We denote by $\boldsymbol{v}$ the peculiar velocities (with the Hubble expansion subtracted), by $\rho$ the normalised matter density, by $\varphi_{\mathrm{g}}$ the gravitational potential and by $\tau$ a suitable time variable (see below). Since, in this paper, we want to concentrate on fluid dynamical aspects, we shall limit ourselves to an Einstein-de Sitter (EdS) model of a flat matter-dominated universe, ignoring the cosmological term responsible for the accelerated expansion of the Universe. For the EdS model the Euler-Poisson equations take the following form (cf. Brenier et al. 2003, and references therein):

$$
\begin{aligned}
\partial_{\tau} \boldsymbol{v}+(\boldsymbol{v} \cdot \nabla) \boldsymbol{v} & =-\frac{3}{2 \tau}\left(\boldsymbol{v}+\nabla \varphi_{\mathrm{g}}\right), \\
\partial_{\tau} \rho+\nabla \cdot(\rho \boldsymbol{v}) & =0 \\
\nabla_{\mathrm{E}}^{2} \varphi_{\mathrm{g}} & =\frac{\rho-1}{\tau}
\end{aligned}
$$

(here $\nabla_{\mathrm{E}}^{2}$ is the Eulerian Laplacian). Eq. (3.2) is the momentum equation, (3.3) expresses mass conservation, (3.4) is the Poisson equation for the gravitational potential. One advantage of the variables here chosen is that such cosmological equations significantly resemble standard fluid mechanical equations.

We comment now on the time variable $\tau$, which is not the cosmic time $t$, but is proportional to $t^{2 / 3}$. Let us linearise the Euler-Poisson equations about the steady state $\boldsymbol{v}=0, \rho=1$. Introducing the density perturbation $\delta \equiv \rho-1$, we obtain from (3.2)-(3.4)

$$
\partial_{\tau \tau}^{2} \delta=-\frac{3}{2 \tau}\left(\partial_{\tau} \delta-\frac{\delta}{\tau}\right)
$$

It is immediately seen that (3.5) has two power-law solutions, $\tau$ and $\tau^{-3 / 2}$. In the former, density perturbations grow linearly with $\tau$, hence the name "linear growth time" for the variable $\tau$. (The standard notation in cosmology for the linear growth time is $D$ or $D(t)$.) The latter mode is decaying at large times but blows up as $\tau \rightarrow 0$, thus invalidating 
linearisation. In the full cosmological setting $\tau$ may not go all the way to zero, because the matter-dominated description used for deriving the Euler-Poisson equations is valid only after the decoupling of matter and radiation. In the cosmic time variable $t$, starting with the Big Bang, this happens around $t=380,000$ years, a kind of boundary layer when compared to the present age of the Universe $t \approx 13.8$ billion years. We shall ignore such effects and let $\tau$ become arbitrarily small. Since $\tau$ appears in the denominator of the right-hand sides of (3.2) and (3.4), if we demand that the solutions remain wellbehaved as $\tau \rightarrow 0$, the initial conditions must satisfy two slaving conditions, $\rho \rightarrow 1$ and $\boldsymbol{v} \rightarrow-\nabla \varphi_{\mathrm{g}}^{(\text {init) }}$ for $\tau \rightarrow 0$. Eq. (3.2) then implies that the velocity remains potential in Eulerian (but not Lagrangian!) coordinates at any $\tau>0$.

\subsubsection{Basic equations in Lagrangian variables}

Fluid dynamicists, since the time of Lagrange, denote what was later called Lagrangian coordinates by the letter $\boldsymbol{a}$. We shall follow this tradition. Cosmologist typically denote it by $\boldsymbol{q}$, to avoid the letter $a$, standing for the expansion scale factor. We, however, avoid the sub- or superscript 0 when referring to initial conditions - in cosmology zero refers to zero redshift, that is the present epoch. Instead, as in Section 2, we use the superscript (init).

To derive the Lagrangian form of the Euler-Poisson equations from (3.2)-(3.4), we shall use a mixture of the methods already employed for the incompressible case, and of a technique widely used in cosmological fluid dynamics (see, e.g., Ehlers \& Buchert 1997).

As in the incompressible case (2.1), because the velocity is potential, the right-hand side of (3.2) is again a Eulerian gradient. Thus we can apply identically the CauchyWeber derivation of Section 2.1. The only difference is that now the initial vorticity vanishes. Thus, (2.7) reduces to

$$
\sum_{k=1}^{3} \nabla^{\mathrm{L}} \dot{x}_{k} \times \nabla^{\mathrm{L}} x_{k}=0 .
$$

A related cubic equation, expressing the vanishing of the current Eulerian vorticity is found in Rampf \& Buchert (2012, cf. eq. (2.24)).

The second equation, replacing the incompressibility condition of the hydrodynamic problem, stems from mass conservation expressed as $\rho J=1$, where $J=\operatorname{det}\left(\nabla^{\mathrm{L}} x\right)$ is the Jacobian of the Lagrangian map. We note that the left-hand side of (3.2) is the acceleration $\ddot{\boldsymbol{x}}$. Taking the Eulerian divergence of (3.2), using (3.4) and $\boldsymbol{v}=\dot{\boldsymbol{x}}$, we obtain

$$
\nabla \cdot\left(\ddot{\boldsymbol{x}}+\frac{3}{2 \tau} \dot{\boldsymbol{x}}\right)=-\frac{3}{2 \tau^{2}} \nabla_{\mathrm{E}}^{2} \varphi_{\mathrm{g}}=-\frac{3}{2 \tau^{2}}(\rho-1) .
$$

Next, we have to reexpress the Eulerian divergence in terms of Lagrangian space derivatives. For this, we need the inverse of the Jacobian matrix. We use the following identity, true for an arbitrary smooth 3D map:

$$
\nabla_{i} a_{j}=\frac{\varepsilon_{j m n} \varepsilon_{i k l}}{2 J} \nabla_{m}^{\mathrm{L}} x_{k} \nabla_{n}^{\mathrm{L}} x_{l} .
$$

(This expression is more convenient than the one involving cofactor matrices.) Eq. (3.7) becomes then

$$
\varepsilon_{j m n} \varepsilon_{i k l} \nabla_{m}^{\mathrm{L}} x_{k} \nabla_{n}^{\mathrm{L}} x_{l}\left(\partial_{\tau \tau}^{2}+\frac{3}{2 \tau} \partial_{\tau}\right) \nabla_{j}^{\mathrm{L}} x_{i}=\frac{3}{\tau^{2}}(J-1) .
$$

Eq. (3.6) and (3.9) constitute a closed system of Lagrangian equations for fluid particle 
motion. The equations, particularly (3.9), may have solutions possibly singular at $\tau=0$ due to the presence of negative powers of $\tau$. Actually, with the slaving conditions (or, equivalently, the absence of the decaying mode), it is easily checked that solutions exist in the form of power series that are non-singular at $\tau=0$. Such expansions in powers of the linear growth time $\tau$ are widely used in the cosmological literature on the Lagrangian perturbation expansion for an EdS universe (see, e.g., Matsubara 2008). The formal power series is constructed in Section 3.2. Convergence and analyticity are then established in Section 3.3.

We also observe that, as in the incompressible case, the system (3.6) and (3.9) is invariant under a change to an arbitrarily accelerated frame. In cosmology this is called "extended Galilean invariance" (see Bernardeau 2013, and references therein).

\subsection{The formal Taylor expansion}

We now seek a solution to (3.6)-(3.9) in the form of a power series in $\tau$ for the displacement $\boldsymbol{\xi} \equiv \boldsymbol{x}-\boldsymbol{a}$ :

$$
\boldsymbol{\xi}(\boldsymbol{a}, t)=\sum_{s=1}^{\infty} \boldsymbol{\xi}^{(s)}(\boldsymbol{a}) \tau^{s} .
$$

Upon substitution of this series into (3.9) we obtain the following relations among the Taylor coefficients (summation over repeated indices is now assumed):

$$
\begin{aligned}
(2 s+3)(s-1) \nabla^{\mathrm{L}} \cdot \boldsymbol{\xi}^{(s)} & =\sum_{n_{1}+n_{2}=s}\left(2 n_{1}^{2}+n_{1}-\frac{3}{2}\right)\left(\nabla_{j}^{\mathrm{L}} \xi_{i}^{\left(n_{2}\right)} \nabla_{i}^{\mathrm{L}} \xi_{j}^{\left(n_{1}\right)}-\nabla_{i}^{\mathrm{L}} \xi_{i}^{\left(n_{2}\right)} \nabla_{j}^{\mathrm{L}} \xi_{j}^{\left(n_{1}\right)}\right) \\
& -\sum_{n_{1}+n_{2}+n_{3}=s}\left(n_{3}^{2}+\frac{n_{3}-1}{2}\right) \varepsilon_{j m n} \varepsilon_{i k l} \nabla_{m}^{\mathrm{L}} \xi_{k}^{\left(n_{1}\right)} \nabla_{n}^{\mathrm{L}} \xi_{l}^{\left(n_{2}\right)} \nabla_{j}^{\mathrm{L}} \xi_{i}^{\left(n_{3}\right)} .
\end{aligned}
$$

This equation is trivially satisfied for $s=1$. For $s>1$, we symmetrise both sums in the right-hand side by performing all possible permutations of indices to obtain

$$
\begin{aligned}
\nabla^{\mathrm{L}} \cdot \boldsymbol{\xi}^{(s)} & =\sum_{0<n<s} \frac{n^{2}+(s-n)^{2}+(s-3) / 2}{s^{2}+(s-3) / 2} \sum_{1 \leq i<j \leq 3}\left(\nabla_{j}^{\mathrm{L}} \xi_{i}^{(s-n)} \nabla_{i}^{\mathrm{L}} \xi_{j}^{(n)}-\nabla_{i}^{\mathrm{L}} \xi_{i}^{(s-n)} \nabla_{j}^{\mathrm{L}} \xi_{j}^{(n)}\right) \\
& -\frac{1}{6} \sum_{n_{1}+n_{2}+n_{3}=s} \frac{n_{1}^{2}+n_{2}^{2}+n_{3}^{2}+(s-3) / 2}{s^{2}+(s-3) / 2} \varepsilon_{j m n} \varepsilon_{i k l} \nabla_{m}^{\mathrm{L}} \xi_{k}^{\left(n_{1}\right)} \nabla_{n}^{\mathrm{L}} \xi_{l}^{\left(n_{2}\right)} \nabla_{j}^{\mathrm{L}} \xi_{i}^{\left(n_{3}\right)} .
\end{aligned}
$$

The other relations, stemming from (3.6), are obtained for $s \geq 1$ just as in Section 2.2:

$$
\nabla^{\mathrm{L}} \times \boldsymbol{\xi}^{(s)}=\frac{1}{2} \sum_{\substack{1 \leq k \leq 3, 0<n<s}} \frac{s-2 n}{s} \nabla^{\mathrm{L}} \xi_{k}^{(n)} \times \nabla^{\mathrm{L}} \xi_{k}^{(s-n)} .
$$

Consequently, for $s \geq 1$ the Taylor coefficients $\boldsymbol{\xi}^{(s)}$ can be determined by performing a Helmholtz-Hodge decomposition (2.14) (now, $\mathbf{R}^{(1)}$ and $R^{(2)}$ denote the right-hand sides of (3.13) and (3.12), respectively).

Observe that the recurrence relations (3.12)-(3.13) for this compressible case turn out to be identical in structure to (2.12)-(2.13) for the incompressible one. This is not surprising given that, in Lagrangian coordinates, the fluid particle displacements have rotational and gradient components in both cases.

In the cosmological literature similar expansions are found, which are not always timeTaylor expansions, but expansions in powers of the magnitude of the displacement. They 
have been carried out up to the fourth order (Rampf \& Buchert 2012). To the best of our knowledge, explicit all-order recurrence relations such as (3.12)-(3.13) have never been presented in the literature. They are essential if we want to investigate the convergence of the expansion and the analyticity of Lagrangian trajectories.

We have checked that our recurrence relations are consistent with the known results up to the fourth order. In particular, the first term $\boldsymbol{\xi}^{(1)}=\nabla^{\mathrm{L}} \varphi_{\mathrm{g}}^{(\mathrm{init})}$ yields the BurgersZeldovich approximation, in which fluid particles keep their initial velocities, often used to initialise numerical simulations. It is important to observe that, in the one-dimensional case when the initial velocity depends only on a single coordinate, the Taylor expansion terminates exactly with the term linear in $\tau$. In other words, in one dimension the Zeldovich approximation is exact (Buchert 1992).

\subsection{Analyticity in time of fluid-particle trajectories}

The Lagrangian perturbation theory in cosmology has been, since its introduction about twenty years ago, a formal expansion in powers of a parameter controlling the amplitude of particle displacements. For the case of an EdS universe, the linear growth time $\tau$ is an appropriate control parameter and the Lagrangian map becomes a formal Taylor series in powers of $\tau$. (Analyticity issues for non-EdS models having for example a cosmological term, are beyond the scope of the present paper.) With the tools developed in Section 2 it is now easy to see that with suitable conditions on the initial velocity field (or, equivalently, on the initial gravitational potential) this formal expansion is actually convergent for small enough $\tau$ and defines an analytic function of $\tau$. Indeed, given the structure of the recurrence relations (3.12)-(3.13), whenever the initial velocity gradient is in any of the function spaces considered in Sections 2.3, 2.4 and 2.5, analyticity will be guaranteed for at least a finite time. To just show analyticity, it suffices to observe that all the coefficients appearing in (3.12)-(3.13) are uniformly bounded by unity. Improved bounds on the radius of convergence of the time-Taylor series can be obtained for the case of an initial velocity gradient with a summable Fourier series just as in Section 2.3.1: all the estimates derived in the incompressible case still hold, without any alterations, in the compressible Euler-Poisson case. Further enhancements, such as discussed in Section 2.6.2 can also be implemented (and actually yield a larger radius of convergence of the Taylor series). For the case of an initial velocity analytic in the space variable, the proof of Section 2.4 that spatio-temporal analyticity persists for at least a finite time holds identically.

Let us illustrate this for the simple case when the initial velocity gradient $\nabla^{2} \varphi_{\mathrm{g}}^{(\text {init })}$ is Hölder-continuous of exponent $\alpha$. As in Section 2.5, we introduce the generating function

$$
\zeta_{\alpha}(\tau) \equiv \sum_{s=1}^{\infty}\left|\nabla^{\mathrm{L}} \boldsymbol{\xi}^{(s)}\right|_{\alpha} \tau^{s}
$$

Applying (2.14), (3.12) and (3.13), we find

$$
6 \Theta_{\alpha} \zeta_{\alpha}^{3}+12 \Theta_{\alpha} \zeta_{\alpha}^{2}-\zeta_{\alpha}+\tilde{\tau} \geq 0
$$

where $\tilde{\tau}=\tau \max _{i j}\left|\nabla_{i}^{\mathrm{L}} \nabla_{j}^{\mathrm{L}} \varphi_{\mathrm{g}}^{(\mathrm{init})}\right|_{\alpha}$. Proceeding as in Frisch \& Zheligovsky (2014), we find that analyticity holds until $\tau_{c}=\mathcal{T} / \max _{i j}\left|\nabla_{i}^{\mathrm{L}} \nabla_{j}^{\mathrm{L}} \varphi_{\mathrm{g}}^{(\text {init })}\right|_{\alpha}$, where for small $\alpha$

$$
\mathcal{T}=\left(48 \Theta_{\alpha}\right)^{-1}+\mathrm{O}\left(\Theta_{\alpha}^{-2}\right) \sim \alpha .
$$

Thus, the smaller the Hölder exponent $\alpha$ of the initial velocity gradient, the smaller is the guaranteed interval of time analyticity. If however the initial velocity gradient, or the initial density fluctuations are not sufficiently smooth, the series may well fail 
to converge. For example, Sahni \& Shandarin (1996) investigated the case of an initial "top-hat underdensity" that is initially discontinuous, and found that low-order perturbation worked better than higher-orders, which they regarded as a possible evidence for "semiconvergence" of the perturbation series.

\section{Concluding remarks}

In this paper we have considered two instances of ideal three-dimensional flow, incompressible flow (Section 2) and a gravity-driven compressible flow of cosmological relevance (Section 3). In both cases, we have shown that the motion of fluid particles is time-analytic, for at least a finite time, when the initial vorticity or velocity gradient, respectively, are slightly better than just continuous in the space variables (for example, Hölder-continuous).

Ours are solutions to the Euler or the Euler-Poisson problems with a Cauchy-type formulation in Lagrangian coordinates. But do they yield solutions to the equations in the original formulations in Eulerian coordinates? The latter are obtained from the former ones by using the global inverse Lagrangian map, whose existence is therefore required for such a construction. For the case of an incompressible flow, the existence of a global inverse Lagrangian map is easily proved using the conservation of volumes. In the compressible Euler-Poisson case, analyticity until some time $\tau_{\star}$ does not imply existence of such a global inverse Lagrangian map. Indeed, in the particular case of one dimension, for which the Burgers-Zeldovich approximation is exact (cf. Section 3.2), the Lagrangian map is a linear function of $\tau$ that is trivially analytic for all $\tau$, but the invertibility holds only until the time of the first crossing of particle paths (called "shell crossing" in cosmology).

What happens to fluid particles beyond the real positive time, say, $t_{1}$, until which our results guarantee analyticity of their trajectories? If the invertibility requirement for the Eulerian formulation does hold till $t_{1}$ and the Eulerian solution is still in the appropriate function space (for example, has a vorticity whose Fourier series is absolutely summable), then we can again obtain analytical trajectories till time $t_{2}>t_{1}$ by constructing a new time-Taylor series, starting at time $t_{1}$ (in the cosmological setting, minor modifications are required, since (3.9) is not autonomous in time). This procedure can be iterated any number of times until one of the conditions stated above is violated and the Eulerian solution becomes therefore rougher than initially.

Does this happen and if so when? For the case of incompressible flow, this is actually the issue of whether a blow up of solutions can occur after a finite time, a major mathematical question. By contrast, for compressible flow in cosmology there is hardly any doubt that such finite-time blow up does occur, essentially because fast particles can catch up with slower ones. In this case, however, a physical question is open: is there an interval of time after the matter-radiation decoupling, during which no particle crossing occurs? This depends very much on what are the small-scale properties of the density and velocity fluctuations at decoupling that are inherited from primordial cosmology, questions that will be addressed elsewhere.

Finally, we want to stress again how remarkably similar are the Lagrangian dynamics of incompressible and compressible ideal flow when tackled in the spirit of Cauchy's 1815 work.

We are grateful to C. Bardos, F. Bernardeau, F. Bouchet, T. Buchert, S. Colombi, J.-P. Kahane, G. Lebeau, H.K. Moffatt, A. Shnirelman, L. Székelyhidi and B. Villone for fruitful discussions. VZ was supported by the grant 11-05-00167-a from the Russian 
foundation for basic research. His visits to the Observatoire de la Côte d'Azur (France) were supported by the French Ministry of Higher Education and Research.

\section{REFERENCES}

AkHiezer, N.I. 1990 Elements of the theory of elliptic functions. AMS Translations of Mathematical Monographs vol. 79. AMS, Rhode Island.

Arfken, G.B. \& Weber, H.J. 2005 Mathematical method for physicists. Elsevier, Amsterdam.

Benachour, S. 1976 Analyticité des solutions périodiques de l'équation d'Euler en trois dimensions. C. R. Acad. Sci. Paris Série A 283, 107-110.

BernardeAu, F. 2013 The evolution of the large-scale structure of the universe: beyond the linear regime. Lectures given to the Les Houches Summer School Post-Planck Cosmology, 8 July - 2 August 2013 http://arxiv.org/abs/1311.2724

Bouchet, F.R., Juszkiewicz, R., Colombi, S. \& Pellat, R. 1992 Weakly nonlinear gravitational instability for arbitrary $\Omega$. Astrophys. J. 394, L5-L8 http://adsabs.harvard. edu/abs/1992ApJ... 394L . . .5B.

Bouchet, F.R., Colombi, S., Hivon, E. \& Juszkiewicz, R. 1995 Perturbative Lagrangian approach to gravitational instability. Astron. Astrophys. 296, 575-608 http://adsabs. harvard.edu/abs/1995A\%26A . . 296. .575B.

Brachet, M.E., Meiron, D.I., Orszag, S.A., Nickel, B.G., Morf, R.H. \& Frisch, U. 1983 Small-scale structure of the Taylor-Green vortex. J. Fluid Mech. 130, 411-452.

Brenier, Y., Frisch, U., Hénon, M., Loeper, G., Matarrese, S., Mohayaee, R. \& SobolevskiI, A. 2003 Reconstruction of the early Universe as a convex optimization problem. Mon. Not. R. Astron. Soc. 346, 501-524. arXiv:astro-ph/0304214.

Buchert, T. 1989 A class of solutions in Newtonian cosmology and the pancake theory Astron. Astrophys. 22, 9-24.

Buchert, T. 1992 Lagrangian theory of gravitational instability of Friedman-Lemaitre cosmologies and the "Zel'dovich approximation" Mon. Not. R. Astron. Soc. 254, 729-737 http://adsabs . harvard. edu/abs/1992MNRAS. 254 . .729B.

Buckmaster, T., De Lellis, C. \& Székelyhidi JR, L. 2013 Transporting microstructure and dissipative Euler flows. arXiv:1302.2815 [math.AP]

CAuchy A.L. 1815 Sur l'état du fluide à une époque quelconque du mouvement. Mémoires extraits des recueils de l'Académie des sciences de l'Institut de France, Théorie de la propagation des ondes à la surface d'un fluide pesant d'une profondeur indéfinie (Extraits des Mémoires présentés par divers savants à l'Académie royale des Sciences de l'Institut de France et imprimés par son ordre). Sciences mathématiques et physiques. Tome I, 1827 Seconde Partie, pp. 33-73. http://gallica.bnf.fr/ark:/12148/bpt6k90181x.r=0euvres+ completes $+\mathrm{d} \% 27$ Augustin+Cauchy . langFR

Chemin J.-Y. 1992 Régularité des trajectoires des particules d'un fluide incompressible remplissant l'espace. J. Mathématiques Pures et Appliquées 71, 407-417.

Constantin, P., Vicol, V. \& Wu, J. 2014 Analyticity of Lagrangian trajectories for wellposed inviscid incompressible fluid models. Submitted. arXiv:1403.5749 [math.AP]

Dombre, T., Frisch, U., Greene, J.M., Hénon, M., Mehr, A. \& Soward, A.M. 1986 Chaotic streamlines and Lagrangian turbulence: the ABC-flows. J. Fluid Mech. 167, 353391.

Ehlers, J. \& BuCherT, T. 1997 Newtonian cosmology in Lagrangian formulation: foundations and perturbation theory. Gen. Rel. Grav. 29, 733-764.

Frisch, U. \& Villone, B. 2014 Cauchy's almost forgotten Lagrangian formulation of the Euler equation for 3D incompressible flow. European Physical J. H, submitted. arXiv:1402.4957 [math.HO].

Frisch, U. \& Zheligovsky, V. 2014 A very smooth ride in a rough sea. Comm. Math. Physics 326, 499-505. arXiv:1212.4333 [math.AP].

Gilbarg, D. \& Trudinger, N.S. 1998 Elliptic partial differential equations of second order. Springer, Berlin.

HANKEL, H. 1861 Zur allgemeinen Theorie der Bewegung der Flüssigkeiten, Preisschrift der philosophischen Facultät der Georgia Augusta, Göttingen. http://babel.hathitrust. org/cgi/pt?id=mdp. 39015035826760 ; view=1up; seq=5 
HÖLDER, E. 1933 Über die unbeschränkte Fortsetzbarkeit einer stetigen ebenen Bewegung in einer unbegrenzten inkompressiblen Flüssigkeit. Mathematische Zeitschrift 37, 727-738.

IsETt, Рн. 2014 Regularity in time along the coarse scale flow for the incompressible Euler equations. arXiv:1307.0565 [math.AP].

Katznelson, Y. 2004 An introduction to harmonic analysis. 3rd ed., Cambridge Univ. Press.

Korn, A. 1907 Sur les équations de l'élasticité. Annales scientifiques de l'É.N.S. $3^{\mathrm{e}}$ série, 24, $9-75$.

KukavicA, I. \& Vicol, V. 2009 On the radius of analyticity of solutions to the threedimensional Euler equations. Proc. Am. Math. Soc. 137, 669-677.

KukAvicA, I. \& Vicol, V. 2011 On the analyticity and Gevrey-class regularity up to the boundary for the Euler equations. Nonlinearity 24, 765-796.

Levermore, C.D. \& Oliver, M. 1997 Analyticity of solutions for a generalized Euler equation. J. Diff. Equations 133, 321-339.

Lichtenstein, L. 1925 Über einige Hilfssätze der Potentialtheorie. I. Mathematische Zeitschrift 23, $72-88$.

Lichtensteın, L. 1927 Über einige Existenzprobleme der Hydrodynamik. Mathematische Zeitschrift 26, 196-323.

Majda, A.J. \& Bertozzi A.L. 2002 Vorticity and incompressible flow. Cambridge Univ. Press.

MatsubarA, T. 2008 Resumming cosmological perturbations via the Lagrangian picture: Oneloop results in real space and in redshift space. Phys. Rev. D 77, 063530.

Moutarde, F., Alimi, J.M., Bouchet, F.R., Pellat, R. \& Raman, A. 1991 Precollapse scale invariance in gravitational instability. Astrophys. J. 382, 377-381.

Pauls, W. \& Matsumoto, T. 2005 Lagrangian singularities of steady two-dimensional flow. Geophys. Astrophys. Fluid Dyn. 99, 61-75.

Pohle, F.V. 1951 The Lagrangian equations of hydrodynamics: solutions which are analytic functions of the time. Thesis, New York University, January 1951.

RAMPF, C. \& BuChERT, T. 2012 Lagrangian perturbations and the matter bispectrum I: fourthorder model for non-linear clustering J. Cosmology and Astrop. Phys. JCAP06(2012)021.

Sahni, V. \& Shandarin, S. 1996 Accuracy of Lagrangian approximations in voids. Mon. Not. R. Astron. Soc. 282, 641-645.

Serfati, Ph. 1992 Étude mathématique de flammes infiniment minces en combustion. Résultats de structure et de régularité pour l'équation d'Euler incompressible. Thèse de Doctorat de l'Université Paris 6.

Serfati, Ph. 1995a Équation d'Euler et holomorphies à faible régularité spatiale. C. R. Acad. Sci. Paris. Série I 320, 175-180.

Serfati, Ph. 1995b Structures holomorphes à faible régularité spatiale en mécanique des fluides. J. Math. Pures Appl. 74, 95-104.

Shnirelman, A. 2012 On the analyticity of particle trajectories in the ideal incompressible fluid. arXiv:1205.5837 [math.AP].

Stein, E.M. 1970 Singular integrals and differentiability properties of functions. Princeton Univ. Press.

StOKeR, J.J. 1957 Water waves: The mathematical theory with applications. Wiley-Interscience.

Stokes, G.G. 1848 Notes on Hydrodynamics. IV. Demonstration of a fundamental theorem. Cambridge and Dublin Mathematical Journal III, 209-219. Available at the Goettingen Archive.

Stokes, G.G. 1883 Notes on Hydrodynamics. IV. Demonstration of a fundamental theorem. In Mathematical and physical papers, vol. II, pp. 36-50. Cambridge Univ. Press.

TAylor, G.I. 1938 The spectrum of turbulence. Proc. R. Soc. A164, 476-490.

TAYlor, G.I. \& GREen, A.E. 1937 Mechanism of the production of small eddies from large ones. Proc. R. Soc. A158, 499-521.

WEBER, H. 1868 Ueber eine Transformation der hydrodynamischen Gleichungen. Journal für die reine und angewandte Mathematik (Crelle) 68, 286-292. Berlin.

Wolibner, W. 1933 Un théorème sur l'existence du mouvement plan d'un fluide parfait, homogène, incompressible, pendant un temps infiniment long. Mathematische Zeitschrift 37, 698-726. 
Yakubovich, E.I. \& Zenkovich D.A. 2001 Matrix approach to Lagrangian fluid dynamics. J. Fluid Mech. 443, 167-196.

Zheligovsky, V. 2011 A priori bounds for Gevrey-Sobolev norms of space-periodic threedimensional solutions to equations of hydrodynamic type. Advances in differential equations 16, 955-976. arXiv:1001.4237 [math.AP]

Zygmund, A. 2002 Trigonometric series. 3rd ed., Cambridge Univ. Press.

\section{Appendix A}

Each step in the derivation of (2.19) is elementary, but their sequence is not necessarily intuitive; this has prompted us to present here the derivation in detail.

Upon substituting the Fourier series (2.17) into (2.13) and collecting the terms involving the exponent $\mathrm{e}^{\mathrm{ip} \cdot \boldsymbol{a}}$ we find

$$
\begin{aligned}
& \mathbf{p} \cdot \widehat{\boldsymbol{\xi}}_{\mathbf{p}}^{(s)}=-\mathrm{i} \sum_{0<m<s, \mathbf{r}} \sum_{1 \leq i<j \leq 3} \varepsilon_{i j k}\left[\mathbf{r}, \mathbf{p}-\mathbf{r}, \mathbf{e}_{k}\right]\left(\widehat{\boldsymbol{\xi}}_{\mathbf{r}}^{(m)}\right)_{i}\left(\widehat{\boldsymbol{\xi}}_{\mathbf{p}-\mathbf{r}}^{(s-m)}\right)_{j} \\
& +\sum_{\substack{n_{1}+n_{2}+n_{3}=s \\
\mathbf{r}_{1}+\mathbf{r}_{2}+\mathbf{r}_{3}=\mathbf{p}}}\left[\mathbf{r}_{1}, \mathbf{r}_{2}, \mathbf{r}_{3}\right]\left(\widehat{\boldsymbol{\xi}}_{\mathbf{r}_{1}}^{\left(n_{1}\right)}\right)_{1}\left(\widehat{\boldsymbol{\xi}}_{\mathbf{r}_{2}}^{\left(n_{2}\right)}\right)_{2}\left(\widehat{\boldsymbol{\xi}}_{\mathbf{r}_{3}}^{\left(n_{3}\right)}\right)_{3},
\end{aligned}
$$

where $\mathbf{e}_{k}$ denotes the $k$-th unit vector of the cartesian coordinate system, $\left(\widehat{\boldsymbol{\xi}}_{\mathbf{r}}\right)_{j}$ the $j$-th component of the vector $\widehat{\boldsymbol{\xi}}_{\mathbf{r}}$, and $k \equiv 6-i-j$. The right-hand side of (A 1$)$ consists of two sums. We replace the first one by the arithmetic mean of this sum and its copy, in which the pairs of indices $(m, \mathbf{r}) \leftrightarrow(s-m, \mathbf{p}-\mathbf{r})$ are swapped. Similarly, we replace the second sum by the arithmetic mean of this sum and its five copies with permuted pairs of indices $\left(n_{i}, \mathbf{r}_{i}\right)$. The replacements involve a group of two terms quadratic in $\widehat{\boldsymbol{\xi}}_{\mathbf{r}_{i}}^{\left(n_{i}\right)}$ and a group of six terms cubic in $\widehat{\boldsymbol{\xi}}_{\mathbf{r}_{i}}^{\left(n_{i}\right)}$; each group readily amalgamates into a triple product, yielding

$$
\begin{aligned}
\mathbf{p} \cdot \widehat{\boldsymbol{\xi}}_{\mathbf{p}}^{(s)}= & -\frac{\mathrm{i}}{2} \sum_{0<m<s, \mathbf{r}} \sum_{k=1}^{3}\left[\mathbf{r}, \mathbf{p}-\mathbf{r}, \mathbf{e}_{k}\right]\left[\widehat{\boldsymbol{\xi}}_{\mathbf{r}}^{(m)}, \widehat{\boldsymbol{\xi}}_{\mathbf{p}-\mathbf{r}}^{(s-m)}, \mathbf{e}_{k}\right] \\
& +\frac{1}{6} \sum_{\substack{n_{1}+n_{2}+n_{3}=s \\
\mathbf{r}_{1}+\mathbf{r}_{2}+\mathbf{r}_{3}=\mathbf{p}}}\left[\mathbf{r}_{1}, \mathbf{r}_{2}, \mathbf{r}_{3}\right]\left[\widehat{\boldsymbol{\xi}}_{\mathbf{r}_{1}}^{\left(n_{1}\right)}, \widehat{\boldsymbol{\xi}}_{\mathbf{r}_{2}}^{\left(n_{2}\right)}, \widehat{\boldsymbol{\xi}}_{\mathbf{r}_{3}}^{\left(n_{3}\right)}\right] .
\end{aligned}
$$

Finally, we note that summation in $k$ in the first sum in (A 2) amounts to calculation of the scalar product; this gives rise to (2.19). 